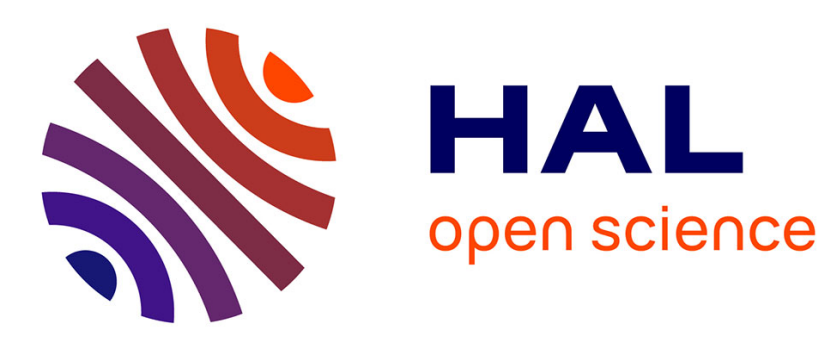

\title{
Concentrations of solutions to time-discretizied compressible Navier-Stokes equations
}

\author{
Pavel Igorevich Plotnikov, Jan Sokolowski
}

\section{To cite this version:}

Pavel Igorevich Plotnikov, Jan Sokolowski. Concentrations of solutions to time-discretizied compressible Navier-Stokes equations. [Research Report] RR-5481, INRIA. 2006, pp.48. inria-00070526

\section{HAL Id: inria-00070526 \\ https://hal.inria.fr/inria-00070526}

Submitted on 19 May 2006

HAL is a multi-disciplinary open access archive for the deposit and dissemination of scientific research documents, whether they are published or not. The documents may come from teaching and research institutions in France or abroad, or from public or private research centers.
L'archive ouverte pluridisciplinaire HAL, est destinée au dépôt et à la diffusion de documents scientifiques de niveau recherche, publiés ou non, émanant des établissements d'enseignement et de recherche français ou étrangers, des laboratoires publics ou privés. 
INSTITUT NATIONAL DE RECHERCHE EN INFORMATIQUE ET EN AUTOMATIQUE

\title{
Concentrations of solutions to time-discretizied compressible Navier-Stokes equations
}

\author{
P. I. Plotnikov — J. Sokolowski
}

\section{$\mathbf{N}^{\circ} \mathbf{5 4 8 1}$}

31 Janvier 2005

Thème NUM

\section{apport}

de recherche 





\title{
Concentrations of solutions to time-discretizied compressible Navier-Stokes equations
}

\author{
P. I. Plotnikov 因, J. Sokolowski 团 \\ Thème NUM — Systèmes numériques \\ Projet Opale \\ Rapport de recherche $n^{\circ} 5481-31$ Janvier 2005 - 48 pages
}

\begin{abstract}
The compactness properties of solutions to time-discretization of compressible Navier-Stokes equations are investigated in three dimensions. The existence of generalized solutions is established.
\end{abstract}

Key-words: Compressible fluids, Navier-Stokes equations, generalized solutions

Mathematics Subject Classification (2000): 35D05, 35Q30, 49Q10, 49Q20, 76N25

* Lavryentyev Institute of Hydrodynamics, Siberian Division of Russian Academy of Sciences, Lavryentyev pr. 15, Novosibirsk 630090, Russia. plotnikov@hydro.nsc.ru

$\dagger$ Institut Elie Cartan, Laboratoire de Mathématiques, Université Henri Poincaré Nancy I, B.P. 239, 54506 Vandoeuvre lès Nancy Cedex, France, Jan.Sokolowski@iecn.u-nancy.fr 


\section{Concentrations des solutions des équations de Navier-Stokes compressible discretisées en temps.}

Résumé : On étudie les propriétés de compacité des solutions des équations de NavierStokes compressible discrétisées en temps en dimension 3. L'existence de solutions généralisées est établie.

Mots-clés : Fluide compressible, équations de Navier-Stokes, solutions généralisées 


\section{Introduction}

Problem formulation A flow of compressible Newtonian fluid in bounded domain $\Omega \subset$ $\mathbb{R}^{3}$ is governed by the Navier-Stokes equations

$$
\begin{aligned}
& \frac{\partial(\varrho \mathbf{u})}{\partial t}+\operatorname{div}(\varrho \mathbf{u} \otimes \mathbf{u})+\nabla p(\varrho)=\varrho \mathbf{F}+\operatorname{div} \Sigma(\mathbf{u}), \\
& \frac{\partial \varrho}{\partial t}+\operatorname{div}(\varrho \mathbf{u})=0,
\end{aligned}
$$

where $p(\varrho)=\varrho^{\gamma}, \varrho$, and $\mathbf{u}$ are pressure, density, and velocity of fluid, $\mathbf{F} \in C(\Omega)^{3}$ is a given vector field, $\Sigma$ is a viscous stress tensor defined by

$$
\Sigma(\mathbf{u})=\left(\nabla \mathbf{u}+\nabla \mathbf{u}^{\top}\right)+\nu \operatorname{div} \mathbf{u} \mathbf{I}, \quad \operatorname{div} \Sigma(\mathbf{u})=\Delta \mathbf{u}+(1+\nu) \nabla \operatorname{div} \mathbf{u},
$$

where $\nu>-2$ is the viscosity ratio. The existence of generalized solutions to initial boundary-value problems for equations (1) was proved by Lions in [19] and by Feireisl, Matušů-Nečasová, Petzeltová, Straškraba in [13] for all $\gamma>9 / 5$. These results were essentially improved by Feireisl, Novotný, and Petzeltová in paper [14], where the existence of solutions was established for all $\gamma>3 / 2$. For the range $\gamma>3 / 2$, the mathematical theory of compressible Navier-Stokes equations is covered in the book by E. Feireisl [15]. Recall that $\gamma=5 / 3$ for monoatomic gases, $\gamma=7 / 5$ for air, and $\gamma=1$ in isothermal case. The problem of the existence of solutions to (1) for $\gamma<3 / 2$ was listed among other unsolved problems of fluid mechanics in [20]. In the paper we consider a boundary-value problem for the stationary equations, obtained by the time discretization of equations (1),

$$
\begin{array}{r}
\alpha \varrho \mathbf{u}+\operatorname{div}(\varrho \mathbf{u} \otimes \mathbf{u})+\nabla p=\varrho \mathbf{F}+\operatorname{div} \Sigma(\mathbf{u}) \text { in } \mathcal{D}^{\prime}(\Omega), \\
\alpha \varrho+\operatorname{div}(\varrho \mathbf{u})=f \text { in } \mathcal{D}^{\prime}(\Omega), \quad u=0 \text { on } \partial \Omega .
\end{array}
$$

Here $\alpha>0$ is a parameter of the time discretization, $f \in C(\Omega)$ is a given non-negative function.

Let $\mathbb{K}$ denote the cone of non-negative functions functions $\varrho \in L^{1}(\Omega)$. A pair $(\varrho, \mathbf{u}) \in$ $\mathbb{K} \times H_{0}^{1,2}(\Omega)$ is said to be a generalized solution to problem (3) if it satisfies the moment and the mass balance equations, which are understood in the sense of distributions.

Notation In the paper the standard notation is used for the function spaces. The space $H^{1, p}(\Omega)$ is the Sobolev space of functions integrable along with the first order generalized derivatives in $L^{p}(\Omega)$ equipped with its natural norm. For $p=2$ we use the notation $H^{1,2}(\Omega)$ rather than $H^{1}(\Omega)$, and for real $m>0$ we denote the Sobolev space of order $m$ by $H^{m, 2}(\Omega)$. $H_{0}^{1,2}(\Omega)$ is the closure of the space $C_{0}^{\infty}(\Omega)$ in the norm of the Sobolev space $H^{1,2}(\Omega)$. We use also the summation convention over repeated indices $i, j=1,2,3$, e.g., $\varphi_{i, j} \xi_{i} \xi_{j}=$ $\sum_{i, j=1}^{3} \varphi_{i, j} \xi_{i} \xi_{j}$. The support of a function $\varphi$ is denoted by $\operatorname{spt} \varphi$. For a vector function $\varphi \in L^{2}(\Omega)^{3}$ its norm is denoted by $\|\varphi\|_{L^{2}(\Omega)}$, the same notation is used for the tensor functions. We denote by $B(x, R) \subset \mathbb{R}^{3}$ the ball of the radius $R$ with the centre at $x$, and 
by $\mathbb{S}^{2}=\partial B(0,1)=\left\{x \in \mathbb{R}^{3} \quad:|x|=1\right\}$ the unit sphere in $\mathbb{R}^{3}$. A function defined as the mapping $G: \Omega \times \mathbb{R}_{\lambda} \mapsto \mathbb{R}$ means $G(x, \lambda) \in \mathbb{R}$ for $x \in \Omega$ and $\lambda \in \mathbb{R}$. For a positive parameter $\varepsilon>0$ we denote e.g., by $\varphi_{\varepsilon} \in B$ the sequence as $\varepsilon \searrow 0$, and will speak about e.g., the strong (norm) convergence, possibly for a subsequence, i.e., $\lim _{\varepsilon} \backslash 0\left\|\varphi_{\varepsilon}-\varphi\right\|_{B}=0$. We can consider as well as the weak convergence or weak star convergence for the sequence $\varphi_{\varepsilon}$ in the function space $B$.

In addition, by $c$ is denoted a generic constant in all estimates given in the paper.

Generalized solutions The local existence and uniqueness theorems for viscous steady compressible flows were proved in pioneering paper by M. Padula 23]. P.L. Lions, cf [19, established the existence result for problem (3) for all $\gamma \geq 5 / 3$ in the case $\alpha>0$ and $\gamma>5 / 3$ in the case $\alpha=0$. Note that these results can be extended to the range $\gamma>3 / 2$ by method developed by E. Feireisl et al in [13, 14. The solvability of problem (3) in two-dimensional case was proved in [19] for all $(\gamma, \alpha) \in[1, \infty) \times(0, \infty) \cup(1, \infty) \times\{0\}$. The limiting case $\gamma=1$, $\alpha=0$ was considered by the authors in $[25$. Recall that the standard energy estimate gives the following bounds for quantities involved in the equations

$$
\begin{gathered}
\left\|\varrho^{\gamma}\right\|_{L^{1}(\Omega)}+\left\|\varrho|\mathbf{u}|^{2}\right\|_{L^{1}(\Omega)}+\|\mathbf{u}\|_{H^{1,2}(\Omega)} \leq C\left(\alpha, \Omega,\|\mathbf{F}\|_{C(\Omega)},\|f\|_{C(\Omega)}\right) \text { for } \gamma \geq 1, \\
\|\varrho \ln (1+\varrho)\|_{L^{1}(\Omega)} \leq C\left(\alpha, \Omega,\|\mathbf{F}\|_{C(\Omega)},\|f\|_{C(\Omega)}\right) \text { for } \gamma=1 .
\end{gathered}
$$

It is easy to see that in the three-dimensional case the energy estimates and embedding theorems guarantee the inclusion $\varrho|\mathbf{u}|^{2} \in L^{s}(\Omega)$ with $s>1$ if and only if $\gamma>3 / 2$. Hence, for $\gamma \leq 3 / 2$ we have only an $L^{1}$ estimate for the density of the kinetic energy. The question is under what conditions will a weak limit of approximate solutions to equations (3) be a solution. If a sequence of approximate solutions $\left(\varrho_{\varepsilon}, \mathbf{u}_{\varepsilon}\right), \varepsilon>0$, to problem (3) satisfies the inequalities (4) with a constant $C$, independent of $\varepsilon$, we can assume, after passing to a subsequence, that

$$
\begin{aligned}
\mathbf{u}_{\varepsilon} & \rightarrow \mathbf{u} \text { weakly in } H_{0}^{1,2}(\Omega), \quad \varrho_{\varepsilon} \rightarrow \varrho \text { weakly in } L^{\gamma}(\Omega), \\
\varrho_{\varepsilon} \mathbf{u}_{\varepsilon} \otimes \mathbf{u}_{\varepsilon} & \rightarrow \mathcal{M} \text { star weakly in the space of Radon measures as } \varepsilon \rightarrow 0,
\end{aligned}
$$

where $\mathcal{M}=\left(\mathcal{M}_{i, j}\right)_{3 \times 3}$ denotes a $3 \times 3$ matrix-valued Radon measure in $\Omega$. In the general case the weak star defect measure $\mathcal{S}=\mathcal{M}-\varrho \mathbf{u} \otimes \mathbf{u} \neq 0$. This leads to the so-called concentration problem, which was widely discussed in the mathematical literature in connection with vortex sheets dynamics, cf. [9, 10, 27]. According to DiPerna and Majda, we say $\Omega_{\text {sing }}$ is a concentration set if $\mathcal{S}(\Omega \backslash V)=0$ for every open $V \supset \Omega_{\text {sing }}$; the cancellation concentration phenomenon is the case when $\operatorname{div} \mathcal{S}=0$. Hence the question is to describe the structure of the defect measure and to find conditions under which it is equal to 0 .

Results We intend to propose a new approach to this problem which is based on the following result on the compactness properties of solutions to the generalized momentum equation. Suppose that the tensor fields $\Pi_{\varepsilon}=\Pi_{\varepsilon}^{(1)}+\Pi_{\varepsilon}^{(2)}$ and the vector-valued functions 
$g_{\varepsilon}$ are defined on bounded domain $\Omega \subset \mathbb{R}^{3}$ and satisfy the conditions

$$
\begin{gathered}
\left\|\Pi_{\varepsilon}^{(1)}\right\|_{L^{q}(\Omega)}+\left\|g_{\varepsilon}\right\|_{L^{1}(\Omega)} \leq c, \\
\Pi_{\varepsilon}^{(1)} \rightarrow \Pi \text { weakly in } L^{q}(\Omega)^{9}, \quad g_{\varepsilon} \rightarrow g \text { weakly in } L^{1}(\Omega)^{3}, \\
\left\|\Pi_{\varepsilon}^{(2)}\right\|_{L^{3 / 2}(\Omega)}+\sup _{B(x, R) \subset \Omega} \sup _{0<r \leq R}\left|S_{\Pi_{\varepsilon}}^{(2)}(x, r, R)\right|=\xi_{\varepsilon}(R) \rightarrow 0 \text { as } \varepsilon \rightarrow 0 .
\end{gathered}
$$

Here the constants $q>3 / 2$ and $c$ is independent of $\varepsilon$, the integral operator $\mathfrak{S}$ is defined by

$$
\mathfrak{S} \Pi(x, r, R)=\int_{r<|x|<R} \frac{1}{|x-y|}(\mathbf{I}-\mathbf{n} \otimes \mathbf{n}): \Pi(y) d y,
$$

where $\mathbf{n}=|x-y|^{-1}(y-x)$.

Suppose also that a sequence $\left(\varrho_{\varepsilon}, \mathbf{u}_{\varepsilon}\right) \in \mathbb{K} \times H_{0}^{1,2}(\Omega)$ satisfies the energy inequality (4a), relations (5), and the equations

$$
\operatorname{div}\left(\varrho_{\varepsilon} \mathbf{u}_{\varepsilon} \otimes \mathbf{u}_{\varepsilon}\right)+\nabla p\left(\varrho_{\varepsilon}\right)=\operatorname{div} \Pi_{\varepsilon}+g_{\varepsilon} \text { in } \mathcal{D}^{\prime}(\Omega) .
$$

We assume that the total energy density satisfies the weak regularity condition

$$
\lim _{r \searrow 0} r^{-1} \int_{B(x, r) \cap \Omega}\left(\varrho_{\varepsilon}\left|\mathbf{u}_{\varepsilon}\right|^{2}+p\left(\varrho_{\varepsilon}\right)\right) d x=0 \text { for all } x \in \Omega .
$$

The first main result of this work is the following theorem.

Theorem 1.1 (i) If $\gamma>1$ and $\alpha>0$, then $\mathcal{M}=\varrho \mathbf{u} \otimes \mathbf{u}$. Moreover, there exists $\kappa>0$ such that for all $\Omega^{\prime} \Subset \Omega$, the sequences $\varrho_{\varepsilon}\left|\mathbf{u}_{\varepsilon}\right|^{2}$ and $\varrho_{\varepsilon}$ are bounded in $L^{1+\kappa}\left(\Omega^{\prime}\right)$ and $L^{\gamma(1+\kappa)}\left(\Omega^{\prime}\right)$, respectively.

(ii) If $\gamma=1$ and $\alpha>0$, then $\mathcal{M}=\varrho \mathbf{u} \otimes \mathbf{u}+\mathcal{S}$, where matrix-valued defect measure $\mathcal{S}$ has the representation

$$
\int_{\Omega} \varphi(x): d \mathcal{S}(x)=\int_{\Omega_{\text {sing }}} \mathbf{s}(x) \otimes \mathbf{s}(x): \varphi(x) \sigma(x) d \mathcal{H}^{1} \text { for all } \varphi \in C_{0}(\Omega)^{9} .
$$

Here $\Omega_{\text {sing }}$ is a Borel set, in which every compact subset is countable $\left(\mathcal{H}^{1}, 1\right)$ rectifiable, $\mathbf{s}(x)$ is the unit tangent vector to $\Omega_{\text {sing }}$ at point $x, \mathcal{H}^{1}$ is the one-dimensional Hausdorff measure, and $\sigma$ is a non-negative, locally bounded function.

Recall that $\Omega_{\text {sing }}$ is countable $\left(\mathcal{H}^{1}, 1\right)$ rectifiable if there is a family of $C^{1}$ one-dimensional manifolds $\Gamma_{k}$ such that $\mathcal{H}^{1}\left(\Omega_{\text {sing }} \backslash \cup_{k} \Gamma_{k}\right)=0$.

Theorem [1.1] leads to new results on a solvability of problem (3). Suppose that $\Omega$ is a bounded domain with $\partial \Omega \in C^{1+\beta}, \beta \in(0,1)$, and consider a family of boundary-value problems, depending on a small positive parameter $\varepsilon$,

$$
\begin{array}{r}
\left(\alpha \varrho+\sqrt{\varepsilon} \varrho^{5}\right) \mathbf{u}+\operatorname{div}(\mathbf{u} \otimes(\varrho \mathbf{u}-\varepsilon \nabla \varrho))+\nabla p=\varrho \mathbf{F}+\operatorname{div} \Sigma(\mathbf{u}) \text { in } \Omega, \\
\alpha \varrho+\sqrt{\varepsilon} \varrho^{5}+\operatorname{div}(\varrho \mathbf{u})-\varepsilon \Delta \varrho=f \text { in } \Omega, \quad u=0, \partial_{n} \varrho=0 \text { on } \partial \Omega .
\end{array}
$$

$\mathrm{RR} \mathrm{n}^{\circ} 5481$ 
Theorem 1.2 For any $\gamma \geq 1, \alpha>0, \mathbf{F} \in C^{\beta}(\Omega)^{3}$, and non-negative $f \in C^{\beta}(\Omega)$ problem (14) has a solution $\mathbf{u}_{\varepsilon} \in C^{2+\beta}(\Omega)^{3}, \varrho_{\varepsilon} \in C^{2+\beta}(\Omega), \varrho_{\varepsilon}>0$. There is a constant $c$ independent of $\varepsilon$ such that

$$
\begin{gathered}
\left\|\varrho_{\varepsilon}\right\|_{L^{\gamma}(\Omega)}+\left\|\mathbf{u}_{\varepsilon}\right\|_{H^{1,2}(\Omega)}+\left\|\varrho_{\varepsilon}\left|\mathbf{u}_{\varepsilon}\right|^{2}\right\|_{L^{1}(\Omega)} \leq c, \\
\varepsilon^{1 / 2}\left\|\varrho_{\varepsilon}^{4+\gamma}\right\|_{L^{1}(\Omega)}+\varepsilon^{1 / 2}\left\|\varrho_{\varepsilon}^{5}\left|\mathbf{u}_{\varepsilon}\right|^{2}\right\|_{L^{1}(\Omega)} \leq c, \\
\varepsilon^{1 / 8}\left\|\varrho_{\varepsilon} \nabla \mathbf{u}_{\varepsilon}\right\|_{L^{8 / 5}(\Omega)}+\varepsilon^{1 / 8}\left\|\varrho_{\varepsilon} \mathbf{u}_{\varepsilon}\right\|_{L^{24 / 7}(\Omega)} \leq c, \\
\varepsilon^{3 / 4}\left\|\nabla \varrho_{\varepsilon}\right\|_{L^{2}(\Omega)}+\varepsilon\left\|\left(1+\varrho_{\varepsilon}\right)^{\gamma-2}\left|\nabla \varrho_{\varepsilon}\right|^{2}\right\|_{L^{1}(\Omega)} \leq c,
\end{gathered}
$$

In the case $\gamma=1$ any solution to problem (14) also satisfies the inequalities

$$
\left\|\varrho_{\varepsilon} \ln \left(1+\varrho_{\varepsilon}\right)\right\|_{L^{1}(\Omega)}+\varepsilon^{1 / 2}\left\|\varrho_{\varepsilon}^{5} \ln \left(1+\varrho_{\varepsilon}\right)\right\|_{L^{1}(\Omega)} \leq c .
$$

This result along with Theorem 1.1]implies.

Theorem 1.3 (i) If $\gamma>1$ and $\alpha>0$, then problem (3) admits at least one weak solution $\varrho \in L^{\gamma}(\Omega), \mathbf{u} \in H_{0}^{1,2}(\Omega)$, satisfying (4a).

(ii) If $\gamma=1$ and $\alpha>0$, then there are $\varrho \in L^{1}(\Omega)$ and $\mathbf{u} \in H_{0}^{1,2}(\Omega)$ which satisfy and the modified momentum balance equation

$$
\alpha \varrho \mathbf{u}+\operatorname{div}(\varrho \mathbf{u} \otimes \mathbf{u})+\nabla \varrho+\operatorname{div} \mathcal{S}=\varrho \mathbf{F}+\operatorname{div} \Sigma \text { in } \mathcal{D}^{\prime}(\Omega),
$$

where the measure-valued tensor $\mathcal{S}$ meets all requirements of Theorem 1.1.

Theorem 1.3 yields the alternative: Either the concentration set is empty or its Hausdorff dimension is equal to one. Whether concentrations are cancelled or a non-trivial singular set really exists is a question which we cannot decide with certainty. Note only that if approximate solutions and a flow region are also invariant under the action of some group $x \rightarrow x^{\prime}$, then a concentration set and a measure density $\theta$ also are invariant under the action of the this group. The precise definition of the concentration set and the measure density are given below. In particular, the velocity field and the pressure are invariant with respect to the shift $x_{3} \rightarrow x_{3}+$ const, in the case of a two-dimensional flow in the plane $\left(x_{1}, x_{2}\right)$. Therefore, in this case $\Omega_{\text {sing }}$ is the union of a countable set of straight lines and $\theta$ is a constant along each of those. From this we conclude that $\operatorname{div} \mathcal{S}=0$ and concentrations are cancelled in agreement with results of [19] and [25]. The same results hold true for axially symmetric flows. On the other hand, the simple examples show that singularities definitely exist for solutions of the pressureless Navier-Stokes equations, which are used in astrophysics. Finally, let us point out that the results of the present paper can be used, in the same way as in [25], to establish the existence of solutions for the associated shape optimization problems.

Mathematical background There are three aspects of our method which deserve brief description. 
1. The first concerns the estimates for the trace of non-negative, measure-valued tensor fields $\mathcal{E}$ with $\operatorname{div} \mathcal{E} \in H_{0}^{1, q}(\Omega)^{*}$, which play the key role in the proof of Theorem 1.1]

2. The second is the observation that the boundedness of the potential $|x|^{-1} * \mu$ of the measure $\mu$ in $\Omega$ implies the boundedness of the embedding $H_{0}^{1,2}(\Omega) \hookrightarrow L^{2}(\Omega, d \mu)$.

3. The third is the use of kinetic formulation of the mass balance equation (3b to obtain the convergence $p\left(\varrho_{\varepsilon}\right) \rightarrow p(\varrho)$.

Now, we can explain the organization of the paper. Sections 25$]$ are devoted to the proof of Theorem 1.1. In section 2 we derive the estimates for non-negative, matrix-valued Radon measure $\mathcal{E}$ satisfying the equation $\operatorname{div}(\mathcal{E}-\Pi)=g$, in which a tensor field $\Pi$ and a vector-valued function $g$ satisfy the assumptions of Theorem 1.1 We show that the measure density

$$
\theta(x)=\lim _{r \rightarrow 0} \frac{1}{r} \int_{B(x, r)} d \mu
$$

of the Radon measure $\mu=\operatorname{Tr} \mathcal{E}$ and the potential

$$
x \mapsto \int_{\Omega}|x-y|^{-1}(d \mu(y)-\mathbf{n} \otimes \mathbf{n}: d \mathcal{E}(y))
$$

are well defined and bounded on every compact subset of $\Omega$. Applying these results to the measure $d \mathcal{E}_{\varepsilon}=\left(\varrho_{\varepsilon} \mathbf{u}_{\varepsilon} \otimes \mathbf{u}_{\varepsilon}+p\left(\varrho_{\varepsilon}\right) \mathbf{I}\right) d x$ we conclude that the Newtonian potentials $|x|^{-1} * p\left(\varrho_{\varepsilon}\right)$ are uniformly bounded on every compact subset of $\Omega$. In the next section we consider the embedding of the Sobolev space into the space of functions, which are square integrable with respect to some measure $\mu$. The main result is that the embedding $H_{0}^{1,2}(\Omega) \hookrightarrow L^{2}(K, d \mu)$, $K \Subset \Omega$, is continuous, if

$$
\omega(t)=\sup _{x \in K} \int_{B(x, t) \cap K}|x-y|^{-1} d \mu(y)<\infty .
$$

Moreover, the embedding is compact provided that $\omega(t) \rightarrow 0$ as $t \rightarrow 0$. These results yield the statement of Theorem 1.1 for $\gamma>1$. The proof of Theorem 1.1] in the case $\gamma=1$ is based on the connection between mathematical theory of compressible fluids and mathematical problems arising in geometric measure theory. The key observation is that the tensor fields $\varrho_{\varepsilon}\left(\mathbf{u}_{\varepsilon} \otimes \mathbf{u}_{\varepsilon}+\mathbf{I}\right)$ converge weakly to a matrix-valued measure $\mathcal{E}$ satisfying the equation $\operatorname{div}(\mathcal{E}-\Pi)=g$ with $\Pi \in L^{2}(\Omega)^{9}$ and $g \in L^{1}(\Omega)^{3}$. The results of section 2 imply the existence of the measure density $\theta(x)=\lim _{r \rightarrow 0} r^{-1} \int_{B(x, r)} d \operatorname{Tr} \mathcal{E}$ at each point $x \in \Omega$. In section 5 we introduce the weak star defect measure $\mathcal{S}=\mathcal{E}\left\lfloor_{\Omega_{\text {sing }}}\right.$, where $\Omega_{\text {sing }}=\{x: \theta(x)>0\}$. Since the linear form $\varphi \mapsto \int_{\Omega} \nabla \varphi: d \mathcal{E}$ can be regarded as the first variation of a onedimensional varifold, the rectifiability of $\Omega_{\text {sing }}$ and representation (6) follow from the classical results of the theory of varifolds, see [4]. The remaining part of the paper is devoted to the existence theory for boundary-value problem (3). In section [6] we show that the statement 
of Theorem 1.3 holds true under the assumption that, for $\gamma>1$, the sequence of solutions $\varrho_{\varepsilon}$ to problem (14) converges almost everywhere in $\Omega$. To obtain this result we apply the technique developed by Lions and Feireisel et al (cf. [19, [12, [14). In section 7 we recall the famous results of P.L. Lions and E. Feireisel et al on compactness properties of the viscous flux and the basic facts from the theory of the oscillations defect measure proposed by E. Feireisl in [12].

The peculiarity of our approach is the systematic use of representations of weak limits $\bar{\varphi}=$ $w-\lim _{\varepsilon \rightarrow 0} \varphi\left(\varrho_{\varepsilon}\right)$, in the form of the Stieltjes integrals $\int_{\mathbb{R}} \varphi(\lambda) d_{\lambda} \Gamma(x, \lambda)$, in which a monotone distribution function $\Gamma(x, \cdot)$ does not depend on $\varphi$ and has the limits $\lim _{\lambda \rightarrow \pm \infty} \Gamma(x, \lambda)=0,1$.

In this setting the strong convergence of $\varrho_{\varepsilon}$ is equivalent to the equality $\Gamma(1-\Gamma)=0$. In section 8, Lemma 8.1] we show that the distribution function satisfies in the strip $\Omega \times \mathbb{R}$ the kinetic equation

$$
\frac{\partial}{\partial \lambda}[(\lambda \alpha+\lambda \operatorname{div} \mathbf{u}(x)-f(x)) \Gamma]-\operatorname{div}(\mathbf{u}(x) \Gamma)=\alpha \Gamma+\frac{\partial}{\partial \lambda}[\lambda M(\Gamma)+m],
$$

in which $m$ is some non-negative Borel measure and $M$ is non-linear integro-differential operator defined by (112). The preference of the method of such a kinetic equation is that equation (17) allows us to tackle problems, in which the strong convergence does not take place; for instance, the problems with fast oscillating data. Note that, in contrast to the theory of conservation laws (cf. [6]), kinetic formulation of problem (3] involves the nonlinear term. Nevertheless, we show that equation (17) can be renormalized, which leads to the identity $\Gamma(1-\Gamma)=0$ and, thus, to the strong convergence of solutions to problem (14).

\section{Tensor fields with integrable divergence}

In this section we obtain the basic estimates for solutions of equations (11). The most significant of them are the bounds for the Newtonian potential $|x|^{-1} * p\left(\varrho_{\varepsilon}\right)$ of the pressure which are given by Proposition 2.3. With further applications in mind we deduce these estimates from general statement on non-negative tensor fields with divergence from $\left(H_{0}^{1,3 / 2}(\Omega)\right)^{*}$. Suppose that $3 \times 3$ matrix-valued finite Radon measure $\mathcal{E}=\left(\mathcal{E}_{i, j}\right)$ is symmetric and nonnegative :

$$
\mathcal{E}_{i, j}=\mathcal{E}_{j, i}, \quad\left\langle\mathcal{E}_{i, j} \varphi_{i}, \varphi_{j}\right\rangle \geq 0 \text { for all } \varphi_{i}, \varphi_{j} \in C_{0}(\Omega), 1 \leq i, j \leq 3 .
$$

In particular, $\mu=\operatorname{Tr} \mathcal{E}$ is a non-negative Radon measure in $\Omega$. Suppose also that there exist a tensor field $\Pi \in L^{3 / 2}(\Omega)^{9}$ and vector-valued function $g \in L^{1}(\Omega)^{3}$ such that for every vector-function $\varphi \in C_{0}^{\infty}(\Omega)^{3}$,

$$
\int_{\Omega} \partial_{j} \varphi_{i}(y) d \mathcal{E}_{i, j}=\int_{\Omega} \partial_{j} \varphi_{i}(y) \Pi_{i, j}(y) d y-\int_{\Omega} \varphi_{i}(y) g_{i}(y) d y .
$$


It is easy to see that the integral identities (18) can be equivalently assembled as the differential equation $\operatorname{div}(\mathcal{E}-\Pi)=g$. The main result of this section is the following proposition.

Proposition 2.1 Suppose that a tensor field $\Pi$ admits the decomposition $\Pi=\Pi^{(1)}+\Pi^{(2)}$, in which $\Pi^{(1)} \in L^{q}(\Omega)^{9}, q>3 / 2$, and

$$
\left\|\Pi^{(2)}\right\|_{L^{3 / 2}(\Omega)}+\sup _{0<r \leq R} \mathfrak{S} \Pi^{(2)}(x, r, R) \leq C_{K}
$$

for all $x$ in a compact $K \Subset \Omega$ and with $R \leq$ dist $(K, \partial \Omega)$. Then there exists a constant $c_{K}$ depending only on $R, C_{K}, \mu(\Omega),\left\|\Pi^{(1)}\right\|_{L^{q}(\Omega)}$, and $\|g\|_{L^{1}(\Omega)}$ such that

$$
\begin{aligned}
& \frac{1}{r} \int_{B(x, r)} d \mu(y)+\lim _{s \rightarrow 0} \int_{s \leq|x-y| \leq r} \frac{1}{|x-y|}(d \mu(y)-\mathbf{n} \otimes \mathbf{n}: d \mathcal{E}(y)) \leq c_{K}, \\
& \frac{1}{r} \int_{B(x, r)} d \mu(y)=\zeta(x, r)+\psi(x, r) \text { for } x \in K \quad \text { and } r \in(0, R] .
\end{aligned}
$$

Here $\zeta(x, r)$ is a function which increases in $r$ and the remainder is bounded $|\psi(x, r)| \leq$ $\Psi(r)+C_{K}$, with

$$
\Psi(r)=c\left\|\Pi^{(1)}\right\|_{L^{q}(\Omega)} r^{2-3 / q}+\sup _{x \in \Omega} \int_{B(x, r) \cap \Omega}|g| d x \rightarrow 0 \quad \text { as } r \rightarrow 0 .
$$

Note that $\mu-\mathbf{n} \otimes \mathbf{n}: \mathcal{E} \geq 0$ in $B(x, R) \backslash\{0\}$.

The proof of Proposition 2.1 is based on the following lemma.

Lemma 2.2 Under the assumptions of Proposition 2.1] for each $x \in K$,

$$
\begin{gathered}
\frac{M_{1}(x, r)}{r}+\int_{r \leq|x-y| \leq R} \frac{1}{|x-y|}(d \mu(y)-\mathbf{n} \otimes \mathbf{n}: d \mathcal{E}(y))+\zeta_{0}(x, r)= \\
\frac{M_{1}(x, R)}{R}-\frac{1}{R} \int_{B(x, R)} \operatorname{Tr} \Pi d y+ \\
\frac{1}{r} \int_{B(x, r)} \operatorname{Tr} \Pi d y+\mathfrak{S} \Pi(x, r, R)-\int_{B(x, R)} k_{r, R}(y-x) d g(y),
\end{gathered}
$$

$\mathrm{RR} \mathrm{n}^{\circ} 5481$ 
where $k_{r, R}(x)=\min \left\{r^{-1},|x|^{-1}\right\}-R^{-1}$ and non-negative, non-increasing in $r$ functions $\zeta_{0}$, $M_{i}$, are defined by

$$
\begin{gathered}
\zeta_{0}(x, r)=\int_{(r, R]} s^{-1} d_{s} M_{0}(x, s)-\int_{(r, R]} s^{-1} M_{0, s}^{\prime}(x, s) d s, \\
M_{0}(x, s)=\int_{B(x, s)} \mathbf{n} \otimes \mathbf{n}: d \mathcal{E}(y), \quad M_{1}(x, s)=\int_{B(x, s)} d \mu(y) .
\end{gathered}
$$

Proof. By abuse of notation we write simply $\zeta_{0}(r), M_{i}(r)$ for $\zeta_{0}(x, r)$ and $M_{i}(x, r)$, respectively. Select positive $s<r$ and $\delta<R-s$ such that $\mu(\partial B(x, s+\delta))=0$ and $M_{j}^{\prime}(s)<\infty$, $j=0,1$. Choose also a sequence of smooth functions $h_{k}$ satisfying the conditions

$$
\begin{array}{r}
h_{k}(t)=1 \text { for } t \leq s, \quad h_{k}(t)=0 \text { for } t \geq s+\delta=0, \quad-2 / \delta \leq h_{k}^{\prime} \leq 0, \\
h_{k}^{\prime}(t) \rightarrow-1 / \delta \text { as } k \rightarrow \infty \text { for each } t \in(s, s+\delta) .
\end{array}
$$

Substituting the vector-valued function $\varphi(y)=h_{k}(|y-x|)(y-x)$ into (18) we derive the identity

$$
\begin{aligned}
\int_{B(x, s+\delta)} h_{k}(|y-x|)(d \mu(y)- & \operatorname{Tr} \Pi d y)+\int_{B(x, s)} h_{k}(|y-x|)(y-x) \cdot g(y) d y+ \\
& \int_{s<|y-x|<s+\delta} h_{k}^{\prime}(|y-x|)|y-x| \mathbf{n} \otimes \mathbf{n}:(d \mathcal{E}(y)-\Pi(y) d y)=0 .
\end{aligned}
$$

Passing to the limit as $k \rightarrow \infty$ and noting that

$$
\lim _{k \rightarrow \infty} h_{k}^{\prime}(|y-x|) \mathbf{n} \otimes \mathbf{n}=-\delta^{-1} \mathbf{n} \otimes \mathbf{n}
$$

everywhere in the annulus $s<|y-x|<s+\delta$ we arrive at the equality

$$
\begin{gathered}
M_{1}(s)-\frac{s}{\delta}\left(M_{0}(s+\delta)-M_{0}(s)\right)=\int_{B(x, s)}(\operatorname{Tr} \Pi-(y-x) g(y)) d y- \\
\frac{s}{\delta} \int_{B(x, s+\delta) \backslash B(x, s)} \mathbf{n} \otimes \mathbf{n}: \Pi d y+\Delta,
\end{gathered}
$$

where

$$
\begin{gathered}
|\Delta| \leq \frac{1}{\delta} \int_{s<|y-x|<s+\delta}(|y-x|-s) \mathbf{n} \otimes \mathbf{n}: d \mathcal{E}(y)+ \\
\int_{s<|y-x|<s+\delta}(d \mu(y)+|\operatorname{Tr} \Pi| d y+|y-x||g(y)| d y) \rightarrow 0 \quad \text { as } \delta \rightarrow 0 .
\end{gathered}
$$

INRIA 
Letting $\delta \rightarrow 0$ we obtain that for almost every $s \in(0, R]$,

$$
M_{1}(s)-s M_{0}^{\prime}(s)=\int_{B(x, s)} \operatorname{Tr} \Pi d y-s \int_{\partial B(x, s)} \mathbf{n} \otimes \mathbf{n}: \Pi d S-\int_{B(x, s)}(y-x) \cdot g(y) d y .
$$

Multiplying both sides by $s^{-2}$ and integrating the result over the interval $(r, R]$ we arrive at

$$
\begin{aligned}
\int_{(r, R]}\left(\frac{M_{1}(s)}{s^{2}}-\frac{M_{0}^{\prime}(s)}{s}\right) d s= & \frac{1}{r} \int_{B(x, r)} \operatorname{Tr} \Pi d y- \\
& \frac{1}{R} \int_{B(x, R)} \operatorname{Tr} \Pi d y+\mathfrak{S} \Pi(x, r, R)-\int_{B(x, R)} k_{r, R}(y-x) d g(y) .
\end{aligned}
$$

It is easy to see that

$$
\begin{aligned}
\int_{(r, R]}\left(s^{-2} M_{1}(s)-s^{-1} M_{0}^{\prime}(s)\right) d s= & \int_{(r, R]} s^{-2} M_{1}(s) d s-\int_{(r, R]} s^{-1} d M_{1}(s)+ \\
& \int_{(r, R]} s^{-1} d\left(M_{1}(s)-M_{0}(s)\right)+\zeta_{0}(x, r),
\end{aligned}
$$

where $d M_{i}$ are the Stieltjes measures generated by the monotone functions $M_{i}, i=0,1$. Integrating by parts the Stieltjes integral in the right-hand side of (24) gives

$$
\int_{(r, R]} s^{-2} M_{1}(s) d s-\int_{(r, R]} s^{-1} d M_{1}(s)=\frac{1}{r} \int_{B(x, r)} d \mu(y)-\frac{1}{R} \int_{B(x, R)} d \mu(y) .
$$

On the other hand, the Fubini's theorem yields the identity

$$
\int_{(r, R]} s^{-1} d\left(M_{1}(s)-M_{0}(s)\right)=\int_{r \leq|x-y| \leq R} \frac{1}{|x-y|}(d \mu(y)-\mathbf{n} \otimes \mathbf{n}: d \mathcal{E}(y)) .
$$

Combining (23)-(26) we obtain (22) and the lemma follows.

We are now in a position to complete the proof of Proposition 2.1 Since $|\mathfrak{S} \Pi(x, r, R)| \leq$ $\int_{B(x, r)}|x-y|^{-1}|\Pi(y)| d y$, the Hölder inequality implies

$$
\begin{gathered}
\frac{1}{s} \int_{B(x, s)}\left|\operatorname{Tr} \Pi^{(1)}\right| d y \leq c s^{2-3 / q}\left\|\Pi^{(1)}\right\|_{L^{q}(\Omega)}, \\
\frac{1}{s} \int_{B(x, s)}\left|\operatorname{Tr} \Pi^{(2)}\right| d y \leq c\left\|\Pi^{(2)}\right\|_{L^{3 / 2}(\Omega)}, \\
\left|\mathfrak{S} \Pi^{(1)}(x, r, R)\right| \leq c R^{2-3 / q}\left\|\Pi^{(1)}\right\|_{L^{q}(\Omega)},
\end{gathered}
$$

$\mathrm{RR} \mathrm{n}^{\circ} 5481$ 
which along with (22) yields (20). Proceeding towards the representation (21) we set

$$
\begin{gathered}
\zeta(x, r)=-\int_{r \leq|x-y| \leq R} \frac{1}{|x-y|}(d \mu(y)-\mathbf{n} \otimes \mathbf{n}: d \mathcal{E}(y))-\zeta_{0}(x, r)- \\
\frac{M_{1}(R)}{R}+\int_{B(x, R)} K(y-x): \Pi d y-\frac{1}{R} \int_{B(x, R)}(y-x) \cdot g(y) d y, \\
\psi(x, r)=\frac{1}{r} \int_{B(x, r)} \operatorname{Tr} \Pi d y-\int_{B(x, r)} K(x-y): \Pi d y+ \\
\int_{B(x, r)}\left(\frac{1}{r}-\frac{1}{|x-y|}\right)(y-x) \cdot g(y) d y,
\end{gathered}
$$

where $K(y-x)=|x-y|^{-1}(\mathbf{I}-\mathbf{n} \otimes \mathbf{n})$. It remains to note that since $|K(x)| \leq c|x|^{-1}$,

$$
\begin{aligned}
|\psi(x, r)| \leq & c \int_{B(x, r)} \frac{\left|\Pi^{(1)}\right| d y}{|y-x|}+\left|\int_{B(x, r)} K(x-y) \Pi^{(2)} d y\right|+\frac{1}{r} \int_{B(x, r)}\left|\operatorname{Tr} \Pi^{(2)}\right| d y+ \\
& \int_{B(x, r)}|g|(y) d y \leq c\left\|\Pi^{(1)}\right\|_{L^{q}(\Omega)} r^{2-3 / q}+\sup _{x \in K} \int_{B(x, r)}|g|(y) d y+C_{K}=\Psi(r)+C_{K},
\end{aligned}
$$

and the proposition follows.

We apply Proposition 2.1 to obtain estimates for the solutions to equations (11). It is easy to see that, under the assumptions of Theorem[1.1, the measures $d \mathcal{E}_{\varepsilon}=\left(\varrho_{\varepsilon} \mathbf{u}_{\varepsilon} \otimes \mathbf{u}_{\varepsilon}+p\left(\varrho_{\varepsilon}\right) \mathbf{I}\right) d y$ and $d \mu_{\varepsilon}=\left(\varrho_{\varepsilon}\left|\mathbf{u}_{\varepsilon}\right|^{2}+3 p\left(\varrho_{\varepsilon}\right)\right) d y$ meet all requirements of Proposition 2.1 which implies the following result.

Proposition 2.3 Under the assumptions of Theorem 1.1 for any $R>0$ there exists a constant $c$, which does not depend on $\varepsilon$, such that

$$
\begin{aligned}
& \frac{1}{r} \int_{B(x, r)} d \mu_{\varepsilon}+\lim _{s \rightarrow 0} \int_{s \leq|x-y| \leq R} \frac{\varrho_{\varepsilon}\left|\mathbf{u}_{\varepsilon}^{t}\right|^{2}+2 p\left(\varrho_{\varepsilon}\right)}{|x-y|} d y \leq c, \\
& \frac{1}{r} \int_{B(x, r)} d \mu_{\varepsilon}=\int_{B(x, r)} \frac{\varrho_{\varepsilon}\left|\mathbf{u}_{\varepsilon}^{t}\right|^{2}+2 p\left(\varrho_{\varepsilon}\right)}{|y-x|} d y+\psi_{\varepsilon}(x, r),
\end{aligned}
$$

for all $r \in(0, R]$, where $\mathbf{u}^{t}=\mathbf{u}-(\mathbf{u} \cdot \mathbf{n}) \mathbf{n}$. The remainder is bounded $\left|\psi_{\varepsilon}\right| \leq \Psi(r)+c \xi_{\varepsilon}(R)$, where $\xi_{\varepsilon}(R)$ defined by (9) and

$$
\Psi(r) \leq c r^{2-3 / q}+\sup _{x \in \Omega} \sup _{\varepsilon>0} \int_{B(x, r) \cap \Omega}\left|g_{\varepsilon}\right| d y \rightarrow 0 \quad \text { as } r \rightarrow 0 .
$$


Proof. Inequality (27) obviously follows from the assumptions of Theorem 1.1 and Proposition 2.1 Fix an arbitrary $s<r<R, R=\operatorname{dist}(x, \partial \Omega)$. Substituting $\mathcal{E}_{\varepsilon}, \mu_{\varepsilon}, \Pi_{\varepsilon}$, and $g_{\varepsilon}$ into the identity (22), noting that $\zeta_{0}=0$ for $\varepsilon>0$, replacing $r$ by $s$ and $R$ by $r$ in the resulting equation we obtain

$$
\begin{gathered}
\frac{1}{r} \int_{B(x, r)} d \mu_{\varepsilon}=\frac{1}{s} \int_{B(x, s)} d \mu_{\varepsilon}+\int_{\substack{s \leq|x-y| \leq r\\
}} \frac{1}{|x-y|}\left(\varrho_{\varepsilon}\left|\mathbf{u}_{\varepsilon}^{t}\right|^{2}+2 p\left(\varrho_{\varepsilon}\right)\right) d y+ \\
A^{1}(x, s, r)+A^{2}(x, s, r)+a(x, s, r),
\end{gathered}
$$

where

$$
\begin{gathered}
A^{i}(x, s, r)=\frac{1}{r} \int_{B(x, r)} \operatorname{Tr} \Pi^{(i)} d y--\frac{1}{s} \int_{B(x, s)} \operatorname{Tr} \Pi_{\varepsilon}^{(i)} d y-\mathfrak{S} \Pi_{\varepsilon}^{(i)}(x, s, r), \\
a(x, s, r)=\int_{B(x, r)} k_{s, r} g_{\varepsilon} d y
\end{gathered}
$$

It follows from (7), (9), and (10) that

$$
\left|A^{1}(x, s, r)+a(x, s, r)\right| \leq \Psi(r), \quad\left|A^{2}(x, s, r)\right| \leq \xi_{\varepsilon}(R) .
$$

Letting $s \rightarrow 0$ in (30) and noting that, by hypothesis of Theorem 1.1

$$
\lim _{s \rightarrow 0} s^{-1} \int_{B(x, s)} d \mu_{\varepsilon}=0 \text { for } x \in \Omega
$$

we obtain identity (28) and the proposition follows.

\section{Embedding theorem}

The objective of this sections is to investigate the inequalities that allow the weighted $L^{2}(\Omega, \varrho d x)$ norm of a function be estimated by the $L^{2}$ norm of its partial derivatives.

Let $\Omega \subset \mathbb{R}^{3}$ be a bounded domain and $\mu$ be a finite non-negative Radon measure in $\Omega$. Without loss of generality we can assume that the measure $\mu$ is extended by 0 outside of $\Omega$. Denote by $\mu_{K}$ a compactly supported, finite Radon measure in $\mathbb{R}^{3}$ such that $\mu_{K}(E)=$ $\mu(E \cap K)$ for every Borel set $E$.

V. Maz'ja established, cf. [21, 22, that the embedding $H^{1,2}(\Omega) \hookrightarrow L^{2}(\Omega, d \mu)$ is continuous if and only if $\mu(E) \leq c$ cap (E) for all Borel sets $E \subset \Omega$. On the other hand, D. Adams , cf. [1, 2], proved that the embedding is continuous if $\left\|(-\Delta)^{1 / 2} \mu_{K}\right\|_{L^{2}\left(\mathbb{R}^{3}\right)}^{2} \leq c \mu(K)$ for each compact $K \subset \mathbb{R}^{3}$. Note that formally we have

$$
\left\|(-\Delta)^{1 / 2} \mu_{K}\right\|_{L^{2}\left(\mathbb{R}^{3}\right)}^{2}=-\int_{\mathbb{R}^{3}}\left(\Delta^{-1} \mu_{K}\right) d \mu_{K} \leq \sup _{\mathbb{R}^{3}}\left|\Delta^{-1} \mu_{K}\right| \mu(K) .
$$

$\mathrm{RR} \mathrm{n}^{\circ} 5481$ 
Hence one can expect that the boundedness of the potential $-\Delta^{-1} \mu$ provides the continuity of the embedding. In this section we develop these arguments and present a simple condition, sufficient to guarantee, that the embedding $H^{1,2}(\Omega) \hookrightarrow L^{2}(\Omega, d \mu)$ is continuous and compact.

Theorem 3.1 Suppose that for some compact $K \Subset \Omega$ and $t \in(0, \operatorname{dist}(K, \partial \Omega))$,

$$
\omega(t)=\sup _{x \in K} \int_{B(x, t) \cap K}|x-y|^{-1} d \mu(y)<\infty .
$$

Then there exists a constant $c_{0}$ depending only on $\Omega$ such that for all $v \in H_{0}^{1,2}(\Omega)$,

$$
\int_{K}|v(y)|^{2} d \mu(y) \leq c_{0}\left(\omega(t)\|v\|_{H^{1,2}(\Omega)}^{2}+\|v\|_{L^{1}(\Omega)}^{2}\left(1+t^{-3}\right)^{2}\right) .
$$

The proof relies on the following lemmas. Introduce the quadratic form $\mathcal{L}: H_{0}^{1,2} \mapsto \mathbb{R}$ defined by $\mathcal{L}(v)=\omega(t)\|\nabla v\|_{L^{2}(\Omega)}^{2}+\left(1+t^{-3}\right)^{2}\|v\|_{L^{1}(\Omega)}^{2}$ and set

$$
\operatorname{Cap}_{\mathcal{L}}(E):=\inf \left\{\mathcal{L}(v): v \in C_{0}^{\infty}(\Omega) \text { and } v(x) \geq 1 \text { for all } x \in E\right\} .
$$

Lemma 3.2 Under the assumptions of Theorem 3.1 there is a constant $c_{1}$ depending only on $\Omega$ such that $\mu_{K}(E) \leq c_{1} C_{a p}(E)$ for all Borel sets $E \subset \Omega$.

Proof. Recall that for any $v \in H_{0}^{1,2}(\Omega)$,

$$
v(x)=\frac{1}{4 \pi} \int_{\Omega} \frac{x-y}{|x-y|^{3}} \cdot \nabla v(y) d y \text { in } \Omega \text { and } v(x)=0 \text { outside of } \Omega .
$$

Choose a decreasing function $\eta \in C^{\infty}(\mathbb{R})$ such that $\eta(s)=0$ for $s<t / 8, \eta(s)=1$ for $s>t / 4$, and $\eta^{\prime}(s)<16 / t$. Now rewrite the last identity in the form

$$
\begin{array}{r}
v(x)=\frac{1}{4 \pi} \int_{\Omega}(1-\eta(|x-y|)) \frac{x-y}{|x-y|^{3}} \cdot \nabla v(y) d y+\frac{1}{4 \pi} \int_{\Omega} \eta(|x-y|) \frac{x-y}{|x-y|^{3}} \cdot \nabla v(y) d y \\
\\
\equiv I_{1}(x)+I_{2}(x) .
\end{array}
$$

It is easy to see that

$$
\begin{gathered}
\int_{E}\left|I_{1}(x)\right| d \mu_{K}(x) \leq c \int_{E \cap K} g_{1} *|\nabla v|(x) d \mu(x)= \\
c \int_{\Omega}|\nabla v|(x)\left\{\int_{K \cap E} g_{1}(y-x) d \mu(y)\right\} d x \leq \\
c\|\nabla v\|_{L^{2}(\Omega)}\left\{\int_{\Omega}\left\{\int_{K \cap E} g_{1}(y-x) d \mu(y)\right\}^{2} d x\right\}^{1 / 2},
\end{gathered}
$$


where $g_{1}(x)=|x|^{-2}$ for $|x| \leq t / 4$ and $g_{1}(x)=0$ otherwise. Since the function $g_{1} * g_{1}(x) \leq$ $c|x|^{-1}$ vanishes for $|x| \geq t$, we have

$$
\begin{array}{r}
\left\{\int_{K \cap E} g_{1}(y-x) d \mu(y)\right\}^{2}=\int_{K \cap E}\left\{\int_{K \cap E} g_{1} * g_{1}(y-x) d \mu(y)\right\} d \mu(x) \leq \\
c \int_{K \cap E}\left\{\int_{\{|x-y| \leq t\} \cap K}|x-y|^{-1} d \mu(y)\right\} d \mu(x) \leq c \omega(t) \mu(E \cap K),
\end{array}
$$

which yields the inequality

$$
\int_{E}\left|I_{1}(x)\right| d \mu_{K}(x) \leq c\|\nabla v\|_{L^{2}(\Omega)} \sqrt{\omega(t)} \sqrt{\mu_{K}(E)} .
$$

On the other hand, since the function $\eta(|y|)|y|^{-3} y$ is smooth and the norm of its gradient is bounded by $c\left(1+t^{-3}\right)$, we have $\left|I_{2}\right| \leq c\left(1+t^{-3}\right)\|v\|_{L^{1}(\Omega)}$. Thus, we get

$$
\int_{E}\left|I_{2}(x)\right| d \mu_{K}(x) \leq c\left(1+t^{-3}\right)\|v\|_{L^{1}(\Omega)} \mu_{K}(E) \leq c\left(1+t^{-3}\right)\|v\|_{L^{1}(\Omega)} \sqrt{\mu_{K}(E)} .
$$

Combining (33) and (34) we conclude that for every Borel set $E \subset \Omega$ and for all functions $v \in H_{0}^{1,2}(\Omega)$,

$$
\int_{E}|v| d \mu_{K} \leq c \sqrt{\mathcal{L}(v) \mu_{K}(E)} .
$$

If $v \geq 1$ on a Borel set $E$, then we have

$$
\mu_{K}(E) \leq \int_{E}|v| d \mu_{K}(x) \leq c \sqrt{\mathcal{L}(v)} \sqrt{\mu_{K}(E)},
$$

and the lemma follows.

The next lemma is a version of a well-known Maz'ja result, see [22].

Lemma 3.3 There exists a positive constant $c$, which depends only on $\Omega$ and does not depend on $t$, such that the inequality

$$
\int_{0}^{\infty} \operatorname{Cap}_{\mathcal{L}}\left(N_{t}\right) d t^{2} \leq c \omega(t)\|\nabla v\|_{L^{2}(\Omega)}^{2} d x+c\left(1+t^{-3}\right)^{2}\left(1+\|v\|_{L^{1}(\Omega)}\|v\|_{L^{2}(\Omega)}^{2}\right)
$$

holds for every function $v \in C_{0}^{\infty}(\Omega)$. Here $N_{t}=\{x \in \Omega:|v(x)| \geq t\}$.

Proof. The proof imitates the Maz'ja proof. We start with the observation that since $\operatorname{Cap}_{\mathcal{L}}\left(N_{t}\right)$ decreases in $t$,

$$
\int_{0}^{\infty} \operatorname{Cap}_{\mathcal{L}}\left(N_{t}\right) d t^{2} \leq 3 \sum_{j=-\infty}^{j=\infty} 2^{2 j} \operatorname{Cap}_{\mathcal{L}}\left(N_{2^{j}}\right)
$$

$\mathrm{RR} \mathrm{n}^{\circ} 5481$ 
Now choose a non-decreasing function $\eta \in C^{\infty}(\mathbb{R})$ such that $\eta(s)=0$ for $s \leq 0, \eta(s)=1$ for $s \geq 1$ and $\eta^{\prime}(s) \leq 2$ for all $s$. Set $v_{j}=\eta\left(2^{1-j}|v(x)|-1\right)$. Note that $v_{j}=0$ when $|v(x)|<2^{j-1}$ and $v_{j}(x)=1$ when $|v(x)| \geq 2^{j}$. Using $v_{j}$ as a test function we get the inequality

$$
\operatorname{Cap}_{\mathcal{L}}\left(N_{2^{j}}\right) \leq \omega(t) \int_{N_{2^{j}-1} \backslash N_{2^{j}}}\left|\nabla v_{j}\right|^{2} d x+c\left(1+t^{-3}\right)^{2}\left(\int_{N_{2^{j}-1}}\left|v_{j}\right| d x\right)^{2} .
$$

Obviously $\left|\nabla v_{j}\right| \leq 2^{2-j}|\nabla v|$. Moreover, if $j \geq 0$ and $x \in N_{2^{j-1}}$, then we have $\left|v_{j}(x)\right| \leq$ $2^{1-j}|v(x)|$. It follows from this and (38) that

$$
\begin{array}{r}
\operatorname{Cap}_{\mathcal{L}}\left(N_{2^{j}}\right) \leq 2^{4-2 j} \omega(t) \int_{N_{2^{j-1} \backslash N_{2^{j}}}}|\nabla v|^{2} d x+c\left(1+t^{-3}\right)^{2} \text { for } j \leq 0, \\
\operatorname{Cap}_{\mathcal{L}}\left(N_{2^{j}}\right) \leq 2^{4-2 j} c \omega(t) \int_{N_{2^{j-1} \backslash N_{2^{j}}}|\nabla v|^{2}+2^{2-2 j} c\left(1+t^{-3}\right)^{2}\left(\int_{N_{2^{j-1}}}|v| d x\right)^{2}}^{\text {for } j \geq 0 .}
\end{array}
$$

Substituting these inequalities into (37) we obtain

$$
\int_{0}^{\infty} \operatorname{Cap}_{\mathcal{L}}\left(N_{t}\right) d t^{2} \leq c \omega(t) \int_{\Omega}|\nabla v|^{2} d x+c\left(1+t^{-3}\right)^{2} \sum_{j \geq 0}\left(\int_{N_{2^{j}-1}}|v| d x\right)^{2} .
$$

Obviously we have

$$
\left(\int_{N_{2^{j-1}}}|v| d x\right)^{2} \leq \operatorname{meas}\left(N_{2^{j-1}}\right) \int_{\Omega}|v|^{2} d x, \text { and meas }\left(N_{2^{j-1}}\right) \leq 2^{1-j} \int_{\Omega}|v| d x,
$$

which implies

$$
\left(\int_{N_{2^{j-1}}}|v| d x\right)^{2} \leq 2^{1-j}\|v\|_{L^{2}(\Omega)}^{2}\|v\|_{L^{1}(\Omega)} .
$$

Combining this inequality with (39) gives (36) and the lemma follows.

We are now in a position to complete the proof of Theorem 3.1. It suffices to prove inequality (31) for functions $v \in C_{0}^{\infty}(\Omega)$ normalized by the condition $\|v\|_{L^{2}(\Omega)}=1$. Since by Lemma 3.2 $\mu_{K}(E) \leq c \operatorname{Cap}_{\mathcal{L}}(E)$ for every compact $K \Subset \Omega$, we have

$$
\int_{K}|v|^{2} d \mu(x) \equiv \int_{0}^{\infty} \mu_{K}\left(N_{t}\right) d t^{2} \leq c \int_{0}^{\infty} \operatorname{Cap}_{\mathcal{L}}\left(N_{t}\right) d t^{2} .
$$

Estimating the right side of this inequality, by an application of (36) from Lemma 3.3 and noting that $\|v\|_{L^{2}(\Omega)}=1,\|v\|_{L^{1}(\Omega)} \leq \sqrt{\operatorname{meas}(\Omega)} \leq c$ we obtain (31), which completes the proof. 


\section{Proof of Theorem 1.1 for $\gamma>1$}

We start with the observation that, by Proposition $\left[2.3\right.$, for any compact $\Omega^{\prime} \Subset \Omega$,

$$
\sup _{x \in \Omega^{\prime}} \int_{\Omega}|x-y|^{-1} p_{\varepsilon}(y) d y \leq C\left(\Omega^{\prime}\right) .
$$

$>$ From this, in view of the identity $p_{\varepsilon}=\varrho_{\varepsilon}^{\gamma}$, and by an application of Theorem 3.1] we obtain the estimate

$$
\left\|\varrho_{\varepsilon}^{\gamma}\left|\mathbf{u}_{\varepsilon}\right|^{2}\right\|_{L^{1}\left(\Omega^{\prime}\right)} \leq c\left(\Omega^{\prime}\right)
$$

which along with the energy estimate (4a) and the embedding $H_{0}^{1,2}(\Omega) \hookrightarrow L^{6}(\Omega)$ gives

$$
\int_{\Omega^{\prime}}\left(\varrho_{\varepsilon}\left|\mathbf{u}_{\varepsilon}\right|^{2}\right)^{1+\kappa} d x \leq\left(\int_{\Omega^{\prime}} \varrho_{\varepsilon}^{\gamma}\left|\mathbf{u}_{\varepsilon}\right|^{2} d x\right)^{(1+\kappa) / \gamma}\left(\int_{\Omega^{\prime}}\left|\mathbf{u}_{\varepsilon}\right|^{6} d x\right)^{(\gamma-\kappa-1) / \gamma} \leq C\left(\Omega^{\prime}\right) .
$$

Here $\kappa=2(\gamma-1) /(2+\gamma)$. On the other hand, equation (11) obviously yields, for $r<3 / 2$, the estimate

$$
\left\|\varrho_{\varepsilon}^{\gamma}\right\|_{L^{r}\left(\Omega^{\prime}\right)} \leq c\left(\Omega^{\prime}\right)\left\|\varrho_{\varepsilon}\left|\mathbf{u}_{\varepsilon}\right|^{2}\right\|_{L^{r}\left(\Omega^{\prime}\right)}+\|\Pi\|_{L^{r}\left(\Omega^{\prime}\right)}+\|g\|_{L^{1}\left(\Omega^{\prime}\right)}+\left\|\varrho_{\varepsilon}^{\gamma}\right\|_{L^{1}\left(\Omega^{\prime}\right)} .
$$

$>$ From this, (4a), and the assumptions of the theorem we conclude that $\left\|\varrho_{\varepsilon}^{\gamma}\right\|_{L^{1+\kappa}\left(\Omega^{\prime}\right)} \leq c$ with $c$ independent of $\varepsilon$ for each $\kappa<\min \{3 / 2,2(\gamma-1) /(2+\gamma)\}$. It remains to note that since $\varrho_{\varepsilon} \rightarrow \varrho$ weakly in $L^{\gamma}(\Omega), \mathbf{u}_{\varepsilon} \rightarrow \mathbf{u}$ a.e. in $\Omega$, and the sequence $\varrho_{\varepsilon}\left|\mathbf{u}_{n}\right|^{2}$ is bounded in $L^{1+\kappa}\left(\Omega^{\prime}\right)$, the sequence $\varrho_{\varepsilon} \mathbf{u}_{\varepsilon} \otimes \mathbf{u}_{\varepsilon}$ converges to $\varrho \mathbf{u} \otimes \mathbf{u}$ weakly in $L^{1+\kappa}\left(\Omega^{\prime}\right)^{9}$.

\section{Proof of Theorem 1.1 for $\gamma=1$}

The proof of Theorem 1.1 for $\gamma=1$ is more delicate. We split the proof into two steps. First, we define the concentration set and show that either it is empty or its Hausdorff dimension is equal to 1 .

Concentration set Let us consider the sequences $\left(\varrho_{\varepsilon}, \mathbf{u}_{\varepsilon}\right)$ and $\Pi_{\varepsilon}, g_{\varepsilon}$ satisfying the hypothesis of Theorem 1.1 Recall that $\varrho_{\varepsilon} \rightarrow \varrho$ weakly in $L^{1}(\Omega)$, and $\varrho_{\varepsilon} \mathbf{u}_{\varepsilon, i} \mathbf{u}_{\varepsilon, j}$ are uniformly bounded in $L^{1}(\Omega)$. After passing to a subsequence we can assume that there exists a $3 \times 3$ matrix-valued Radon measure $\mathcal{E}$ such that $\varrho_{\varepsilon} \mathbf{u}_{\varepsilon} \otimes \mathbf{u}_{\varepsilon}+\varrho_{\varepsilon} \mathbf{I} \rightarrow \mathcal{E}$ star weakly in the space of Radon measures on $\Omega$. Obviously the measure $\mathcal{E}$ and its trace $\mu$ meet all requirements of Proposition 2.1] with $\Pi^{(1)}=\Pi \in L^{q}(\Omega), \Pi^{(2)}=0, g=\varrho \mathbf{F}$, and $C_{K}=0$. It follows from Proposition 2.1 that $r^{-1} \mu(B(x, r))=\zeta(x, r)+\psi(x, r)$, where $\zeta(x, r)$ increases in $r$ and $|\psi(x, r)| \leq \Psi(r) \rightarrow 0$ as $r \rightarrow 0$. Hence the Borel function $\theta(x):=\lim _{r \rightarrow 0} r^{-1} \mu(B(x, r))$ is well-defined and bounded on every compact subset of $\Omega$. Split $\Omega$ into two disjoint parts $\Omega_{\text {reg }}$ and $\Omega_{\text {sing }}$ given by

$$
\Omega_{r e g}=\{x: x \in \Omega, \theta(x)=0\}, \quad \Omega_{\text {sing }}=\{x: x \in \Omega, \theta(x)>0\} .
$$


The following theorem shows that $\Omega_{\text {sing }}$ is a concentration set for the sequence $\varrho_{\varepsilon} \mathbf{u}_{\varepsilon} \otimes \mathbf{u}_{\varepsilon}$.

Theorem 5.1 Under the assumptions of Theorem 1.1, for every matrix-valued function $\varphi \in C_{0}(\Omega)^{9}$ we have

$$
\begin{gathered}
\int_{\Omega} \varphi: d \mathcal{E}:=\lim _{\varepsilon \rightarrow 0} \int_{\Omega} \varrho_{\varepsilon}\left(\mathbf{u}_{\varepsilon} \otimes \mathbf{u}_{\varepsilon}+\mathbf{I}\right): \varphi d y= \\
\int_{\Omega_{r e g}} \varrho(\mathbf{u} \otimes \mathbf{u}+\mathbf{I}): \varphi d y+\int_{\Omega_{\text {sing }}} \varphi: d \mathcal{E} .
\end{gathered}
$$

We divide the proof into a sequence of lemmas. In the sequel the notation $K_{\delta}=$ $\{x \mid \operatorname{dist}(x, K) \leq \delta\}$ stands for the compact $\delta$-vicinity of a compact $K \Subset \Omega$.

Lemma 5.2 If a compact $K \subset \Omega_{\text {reg }}$, then for any $\eta>0$ there exist positive $\delta(\eta)$ and $c(\eta)$ such that the inequality

$$
\int_{K_{\delta}} \varrho_{\varepsilon}|v|^{2} \leq \eta\|v\|_{H^{1,2}(\Omega)}^{2}+c(\eta)\|v\|_{L^{2}(\Omega)}^{2}
$$

holds for all $v \in H^{1,2}(\Omega)$ and for any $\delta \leq \delta(\eta)$. Here the constants $\delta(\eta), c(\eta)$ depend on $K$, $\eta$ and do not depend on $\varepsilon$ and $v$.

Proof. First we show that there exist positive $\delta(\eta)$ and $t(\eta)$ such that

$$
\sup _{x \in K_{\delta(\eta)}} \sup _{\varepsilon} \int_{|y-x|<t(\eta)}|y-x|^{-1} \varrho_{\varepsilon}(y) d y \leq \eta .
$$

If the assertion is false, then there are $k>0$ and sequences $x_{\varepsilon} \in \Omega, t_{\varepsilon}>0$, such that

$$
\lim _{\varepsilon \rightarrow 0} \operatorname{dist}\left(x_{\varepsilon}, K\right)=\lim _{\varepsilon \rightarrow 0} t_{\varepsilon}=0 \text { and } \int_{|y-x|<t_{\varepsilon}}|x-y|^{-1} \varrho_{\varepsilon}(y) d y \geq k .
$$

Set $d \mu_{\varepsilon}(x)=\varrho_{\varepsilon}\left(\left|\mathbf{u}_{\varepsilon}\right|^{2}+3\right) d x, R=2^{-1}$ dist $(K, \partial \Omega)$, and recall that, by Proposition 2.3

$$
\int_{B\left(x, t_{\varepsilon}\right)}|y-x|^{-1} \varrho_{\varepsilon}(y) d y \leq \frac{1}{t_{\varepsilon}} \int_{B\left(x_{\varepsilon}, t_{\varepsilon}\right)} d \mu_{\varepsilon}(y)-\psi_{\varepsilon}\left(x_{\varepsilon}, t_{\varepsilon}\right), \text { where }\left|\psi_{\varepsilon}(x, t)\right| \leq \Psi(t)+\xi_{\varepsilon}(R) .
$$

Since, by (9) and (29), $\Psi\left(t_{\varepsilon}\right)+\xi_{\varepsilon}(R) \rightarrow 0$ for $\varepsilon \searrow 0$, the inequality $\mu_{\varepsilon}\left(B\left(x_{\varepsilon}, t_{\varepsilon}\right)>t_{\varepsilon} k / 2\right.$ holds for all sufficiently small $\varepsilon$. After passing to a subsequence we can assume that $x_{\varepsilon}$ converge to some $x \in K$ as $\varepsilon \rightarrow 0$, and hence

$$
\frac{1}{2 t_{\varepsilon}} \int_{B\left(x, 2 t_{\varepsilon}\right)} d \mu_{\varepsilon}(y)>k / 4>0 \text { for all sufficiently small } \varepsilon \text {. }
$$


On the other hand, Proposition 2.3 implies the representation $\mu_{\varepsilon}(B(x, t))=t \zeta_{\varepsilon}(x, t)+$ $t \psi_{\varepsilon}(x, t)$, in which the function $\zeta_{\varepsilon}$ is non-decreasing in $t$. From this and (44) we conclude that for all $t>2 t_{\varepsilon}$,

$$
\frac{k}{4}<\frac{1}{2 t_{\varepsilon}} \int_{B\left(x, 2 t_{\varepsilon}\right)} d \mu_{\varepsilon}(y) \leq \frac{1}{t} \int_{B(x, t)} d \mu_{\varepsilon}(y)+\Psi(t)+\Psi(2 t)+2 \xi_{\varepsilon}(R) .
$$

Note that $\mu_{\varepsilon}(B(x, t)) \rightarrow \mu(B(x, t))$ for almost every positive $t$. Letting $\varepsilon \rightarrow 0$ in the last inequality and noting that $\xi_{\varepsilon} \rightarrow 0$ by Proposition 2.3 we conclude that $k \leq 4 t^{-1} \mu(B(x, t))+$ $4 \Psi(t)+4 \Psi(2 t)$ for almost every positive $t$. Therefore, $\theta(x) \geq k / 4$ which contradicts to the inclusion $x \in \Omega_{r e g}$, and the assertion follows. Now inequality (43) implies that the measures $d \mu_{\varepsilon}=\varrho_{\varepsilon} d x$ satisfy the hypothesis of Theorem 3.1 with $K=K_{\delta(\eta)}, t=t(\eta)$, and $\omega(t)=\eta$. Applying this theorem we obtain (42) which completes the proof.

Lemma 5.3 For any compact $K \Subset \Omega, \varphi \in C(\Omega)$, and $f, g \in H_{0}^{1,2}(\Omega)$,

$$
\lim _{\varepsilon \rightarrow 0} \int_{K} \varphi\left(\varrho_{\varepsilon}-\varrho\right) f g d x=0 .
$$

Proof. Fix $\delta>0$ and choose $\bar{f}, \bar{g} \in C_{0}^{\infty}(\Omega)$ such that

$$
\|f-\bar{f}\|_{H^{1,2}(\Omega)}+\|g-\bar{g}\|_{H^{1,2}(\Omega)}<\delta .
$$

Since the Newtonian potentials of $\varrho$ and $\varrho_{\varepsilon}$ are uniformly bounded on $K$, Theorem 3.1yields the inequality

$$
\int_{K}\left(\varrho_{\varepsilon}+\varrho\right)\left|u\|v \mid d x \leq c\| v\left\|_{H^{1,2}(\Omega)}\right\| u \|_{H^{1,2}(\Omega)} \text { for all } u, v \in H_{0}^{1,2}(\Omega) .\right.
$$

$>$ From this we conclude that

$$
\begin{gathered}
\limsup _{\varepsilon \rightarrow 0}\left|\int_{K} \varphi\left(\varrho_{\varepsilon}-\varrho\right) f g d x\right| \leq \limsup _{\varepsilon \rightarrow 0}\left|\int_{K} \varphi\left(\varrho_{\varepsilon}-\varrho\right) \bar{f} \bar{g} d x\right|+ \\
c(\varphi) \underset{\varepsilon \rightarrow 0}{\limsup } \int_{K}\left(\varrho_{\varepsilon}+\varrho\right)(|g||f-\bar{f}|+|\bar{f}||g-\bar{g}|) d x \leq \\
\quad c \delta\left(\|f\|_{H^{1,2}(\Omega)}+\|g\|_{H^{1,2}(\Omega)}\right) \rightarrow 0 \text { as } \delta \rightarrow 0,
\end{gathered}
$$

which proves the lemma.

Lemma 5.4 Under the assumptions of Theorem 5.1 for any $\eta>0$, a compact $K \Subset \Omega$, and $\varphi \in C(\Omega)$, there exists $\delta(\eta)>0$ such that

$$
\limsup _{\varepsilon \rightarrow 0}\left|\int_{K_{\delta}} \varphi \varrho_{\varepsilon}\left(\left|\mathbf{u}_{\varepsilon}\right|^{2}-|\mathbf{u}|^{2}\right) d x\right| \leq \eta \text { for all } \delta \leq \delta(\eta) .
$$


Proof. Note that for every $\Delta \geq 0$,

$$
\begin{array}{r}
\left|\int_{K_{\Delta}} \varphi \varrho_{\varepsilon}\left(\left|\mathbf{u}_{\varepsilon}\right|^{2}-|\mathbf{u}|^{2}\right) d x\right| \leq c(\varphi)\left(\frac{1}{\sqrt{\eta}} \int_{K_{\Delta}} \varrho_{\varepsilon}\left|\mathbf{u}_{\varepsilon}-\mathbf{u}\right|^{2} d x+\sqrt{\eta} \int_{K_{\Delta}} \varrho_{\varepsilon}\left|\mathbf{u}_{\varepsilon}+\mathbf{u}\right|^{2} d x\right) \leq \\
c \sqrt{\eta}+c \frac{1}{\sqrt{\eta}} \int_{K_{\Delta}} \varrho_{\varepsilon}\left|\mathbf{u}_{\varepsilon}-\mathbf{u}\right|^{2} d x,
\end{array}
$$

where the constant $c$ depends on $\varphi$ and $K$. Set $\Delta=\delta(\eta)$, where $\delta(\eta)$ is defined in Lemma 5.2 Inequality (42) now implies

$$
\int_{K_{\delta(\eta)}} \varrho_{\varepsilon}\left|\mathbf{u}_{\varepsilon}-\mathbf{u}\right|^{2} \leq \eta\left\|\mathbf{u}_{\varepsilon}-\mathbf{u}\right\|_{H^{1,2}(\Omega)}^{2}+c(\eta)\left\|\mathbf{u}_{\varepsilon}-\mathbf{u}\right\|_{L^{2}(\Omega)}^{2} .
$$

Substituting this estimate into (46) we finally obtain

$$
\limsup _{\varepsilon \rightarrow 0}\left|\int_{K_{\delta(\eta)}} \varphi \varrho_{\varepsilon}\left(\left|\mathbf{u}_{\varepsilon}\right|^{2}-|\mathbf{u}|^{2}\right) d x\right| \leq c \sqrt{\eta}+\limsup _{\varepsilon \rightarrow 0} \frac{c(\eta)}{\sqrt{\eta}}\left\|\mathbf{u}_{\varepsilon}-\mathbf{u}\right\|_{L^{2}(\Omega)}^{2}=c \sqrt{\eta},
$$

which completes the proof.

Lemma 5.5 The equality

$$
\int_{K} \varphi d \mu=\int_{K} \varphi\left(|\mathbf{u}|^{2}+3\right) \varrho d y
$$

holds for all compacts $K \subset \Omega_{\text {reg }}$ and all functions $\varphi \in C_{0}(\Omega)$.

Proof. It suffices to prove the lemma for $\varphi \geq 0$. Choose an arbitrary non-negative $\varphi \in C_{0}(\Omega)$. In this case Lemma 5.4 implies the inequalities

$$
\begin{gathered}
\int_{K_{\delta(\eta)}} \varphi\left(|\mathbf{u}|^{2}+3\right) \varrho d y-\eta \leq \liminf _{\varepsilon \rightarrow 0} \int_{K_{\delta(\eta)}} \varphi\left(\left|\mathbf{u}_{\varepsilon}\right|^{2}+3\right) \varrho_{\varepsilon} d y \leq \\
\limsup _{\varepsilon \rightarrow 0} \int_{K_{\delta(\eta)}} \varphi\left(\left|\mathbf{u}_{\varepsilon}\right|^{2}+3\right) \varrho_{\varepsilon} d y \leq \int_{K_{\delta(\eta)}} \varphi\left(|\mathbf{u}|^{2}+3\right) \varrho d y+\eta .
\end{gathered}
$$

On the other hand, since $\varrho_{\varepsilon}\left(\left|\mathbf{u}_{\varepsilon}\right|^{2}+3\right)$ converges star weakly to $\mu$ in the space of the Radon measures on $\Omega$ and $K_{\delta(\eta)}$ is a compact, we have

$$
\begin{gathered}
\int_{\operatorname{int}\left(K_{\delta(\eta)}\right)} \varphi d \mu \leq \liminf _{\varepsilon \rightarrow 0} \int_{\operatorname{int}\left(K_{\delta(\eta)}\right)} \varphi\left(\left|\mathbf{u}_{\varepsilon}\right|^{2}+3\right) \varrho_{\varepsilon} d y \leq \\
\limsup _{\varepsilon \rightarrow 0} \int_{K_{\delta(\eta)}} \varphi\left(\left|\mathbf{u}_{\varepsilon}\right|^{2}+3\right) \varrho_{\varepsilon} d y \leq \int_{K_{\delta(\eta)}} \varphi d \mu .
\end{gathered}
$$


$>$ From this and (48) we conclude that

$$
\int_{\operatorname{int}\left(K_{\delta(\eta)}\right)} \varphi d \mu \leq \int_{K_{\delta(\eta)}} \varphi\left(|\mathbf{u}|^{2}+3\right) \varrho d y+\eta \text { and } \int_{K_{\delta(\eta)}} \varphi d \mu \geq \int_{K_{\delta(\eta)}} \varphi\left(|\mathbf{u}|^{2}+3\right) \varrho d y-\eta \text {. }
$$

Since $\cap_{\eta>0} \operatorname{int}\left(K_{\delta(\eta)}\right)=\cap_{\eta>0} K_{\delta(\eta)}=K$, we have

$$
\lim _{\eta \rightarrow 0} \int_{\operatorname{int}\left(K_{\delta(\eta)}\right)} \varphi d \mu=\lim _{\eta \rightarrow 0} \int_{K_{\delta(\eta)}} \varphi d \mu=\int_{K} \varphi d \mu .
$$

Passing to the limit in (49) as $\eta \rightarrow 0$ we obtain (47), which completes the proof.

Lemma 5.6 The inequality

$$
\int_{K}(\varrho \mathbf{u} \otimes \mathbf{u}+\varrho \mathbf{I}): \varphi \otimes \varphi d x \leq \int_{K} \varphi \otimes \varphi: d \mathcal{E}
$$

holds for every compact set $K \Subset \Omega$ and all vector-valued functions $\varphi \in C_{0}(\Omega)^{3}$.

Proof. Recall that the sequence $\mathbf{u}_{\varepsilon}$ converges to $\mathbf{u}$ almost everywhere in $\Omega$. By the Egoroff's theorem, there exists a sequence of measurable sets $A_{k} \subset K$ such that $\mathbf{u}_{\varepsilon} \rightarrow \mathbf{u}$ uniformly on each $A_{k}$ and meas $\left(K \backslash A_{k}\right) \rightarrow 0$ as $k \rightarrow \infty$. Lemma 5.3 and weak $L^{1}$ convergence of $\varrho_{\varepsilon}$ to $\varrho$ imply the relations

$$
\lim _{\varepsilon \rightarrow 0} \int_{K}\left(\varrho_{\varepsilon}-\varrho\right) \mathbf{u} \otimes \mathbf{u}: \varphi \otimes \varphi d y=0, \quad \lim _{\varepsilon \rightarrow 0} \int_{A_{k}} \varrho_{\varepsilon}\left(\mathbf{u}_{\varepsilon} \otimes \mathbf{u}_{\varepsilon}-\mathbf{u} \otimes \mathbf{u}\right): \varphi \otimes \varphi d y=0,
$$

which along with the inequality $\mathbf{u}_{\varepsilon} \otimes \mathbf{u}_{\varepsilon}: \varphi \otimes \varphi \geq 0$ yields

$$
\begin{aligned}
\limsup _{\varepsilon \rightarrow 0} \int_{K}\left(\varrho_{\varepsilon} \mathbf{u}_{\varepsilon} \otimes \mathbf{u}_{\varepsilon}+\varrho_{\varepsilon} \mathbf{I}\right): \varphi \otimes \varphi d y-\int_{K}(\varrho \mathbf{u} \otimes \mathbf{u}+\varrho \mathbf{I}): \varphi \otimes \varphi d y= \\
\quad \limsup _{\varepsilon \rightarrow 0} \int_{K \backslash A_{k}} \varrho_{\varepsilon}\left(\mathbf{u}_{\varepsilon} \otimes \mathbf{u}_{\varepsilon}-\mathbf{u} \otimes \mathbf{u}\right): \varphi \otimes \varphi d y \geq-\int_{K \backslash A_{k}} \varrho \mathbf{u} \otimes \mathbf{u}: \varphi \otimes \varphi d y
\end{aligned}
$$

Since $\varrho|\mathbf{u}|^{2}$ belongs to $L^{1}(\Omega)$, the last term tends to 0 as $k \rightarrow \infty$, which implies

$$
\limsup _{\varepsilon \rightarrow 0} \int_{K}\left(\varrho_{\varepsilon} \mathbf{u}_{\varepsilon} \otimes \mathbf{u}_{\varepsilon}+\varrho_{\varepsilon} \mathbf{I}\right): \varphi \otimes \varphi d y-\int_{K}(\varrho \mathbf{u} \otimes \mathbf{u}+\varrho \mathbf{I}): \varphi \otimes \varphi d y \geq 0
$$

Since the non-negative $L^{1}$-functions $\left(\varrho_{\varepsilon} \mathbf{u}_{\varepsilon} \otimes \mathbf{u}_{\varepsilon}+\varrho_{\varepsilon} \mathbf{I}\right): \varphi \otimes \varphi$ converge to $\varphi \otimes \varphi: \mathcal{E}$ star weakly in the space of Radon measures on $\Omega$, we have

$$
\limsup _{\varepsilon \rightarrow 0} \int_{K}\left(\varrho_{\varepsilon} \mathbf{u}_{\varepsilon} \otimes \mathbf{u}_{\varepsilon}+3 \varrho_{\varepsilon} \mathbf{I}\right): \varphi \otimes \varphi d y \leq \int_{K} \varphi \otimes \varphi: d \mathcal{E},
$$

$\mathrm{RR} \mathrm{n}^{\circ} 5481$ 
which along with (52) gives (50), and the lemma follows.

We are now in a position to complete the proof of Theorem [5.1 Choose an arbitrary compact $K \subset \Omega_{r e g}$, a non-negative function $h \in C_{0}(\Omega)$, and a vector $\xi \in \mathbb{R}^{3}$. Set $\varphi(x)=$ $\sqrt{h(x)} \xi$. Applying Lemma [5.6 we get the inequality

$$
B \xi \cdot \xi \equiv \int_{K} \varphi \otimes \varphi: d \mathcal{E}-\int_{K}(\varrho \mathbf{u} \otimes \mathbf{u}+\varrho \mathbf{I}): \varphi \otimes \varphi d x \geq 0,
$$

where the symmetric matrix $B$ is given by

$$
B=\int_{K} h d \mathcal{E}-\int_{K} h(\varrho \mathbf{u} \otimes \mathbf{u}+\varrho \mathbf{I}) d x .
$$

By Lemma [5.5, $\operatorname{Tr} B=0$, which implies $B=0$. Hence the equality

$$
\int_{K} h\left(\varrho \mathbf{u}_{i} \mathbf{u}_{j}+\varrho \delta_{i, j}\right) d x=\int_{K} h d \mathcal{E}_{i, j}
$$

holds for all $h \in C_{0}(\Omega)$. Next, choose an increasing sequence $\left\{K_{m}\right\}_{m>1}$ of compact subsets of $\Omega$ such that $\mu\left(\Omega_{r e g} \backslash \cup K_{m}\right)=$ meas $\left(\Omega_{r e g} \backslash \cup K_{m}\right)=0$. From (53) we conclude that for every matrix-valued function $\varphi \in C_{0}(\Omega)^{9}$,

$$
\begin{gathered}
\int_{\Omega_{r e g}} \varphi: d \mathcal{E}=\lim _{m \rightarrow \infty} \int_{K_{m}} \varphi: d \mathcal{E}= \\
\lim _{m \rightarrow \infty} \int_{K_{m}} \varphi:(\varrho \mathbf{u} \otimes \mathbf{u}+\varrho \mathbf{I}) d x=\int_{\Omega_{r e g}} \varphi:(\varrho \mathbf{u} \otimes \mathbf{u}+\varrho \mathbf{I}) d x,
\end{gathered}
$$

and the theorem follows.

Rectifiability of concentration set In this paragraph we show that the set $\Omega_{\text {sing }}$ is countably $\mathcal{H}^{1}$ rectifiable, and investigate in more details the structure of the measure $\mathcal{E}$. Recall some basic facts from geometric measure theory which will be used throughout this section, cf. 11, 4 .

Let $\mu$ be a non-negative Radon measure in $\Omega$ and $a \in \Omega$. Then $\operatorname{VarTan}(\mu, a)$ is a set of those Radon measures $v$ on $\mathbb{R}^{3}$ for which there is a sequence $\left\{\varepsilon_{i}\right\}_{i \geq 1}$ of positive real numbers such that $\lim _{i \rightarrow \infty} \varepsilon_{i}=0$ and

$$
\int_{\mathbb{R}^{3}} \varphi d v=\lim _{i \rightarrow \infty} \varepsilon_{i}{ }^{-1} \int_{\Omega} \varphi\left(\varepsilon_{i}^{-1}(x-a)\right) d \mu .
$$


If there exists $\theta(a)=\lim _{r \rightarrow 0} r^{-1} \mu(B(a, r))<\infty$, then $\operatorname{VarTan}(\mu, a)$ is non-empty, and $v(B(0, r))=$ $r v(B(0,1))=r \theta(a)$ for every member $v$ of $\operatorname{VarTan}(\mu, a)$. The tangential cone to a measure $\mu$ at the point $a$ is the set

$$
\operatorname{Tan}(\mu, a)=\bigcap_{\mathcal{A}}\left\{\operatorname{Tan}(\mathcal{A}, a): \mathcal{A} \subset \Omega \text { and } \lim _{r \rightarrow 0} r^{-1} \mu((\Omega \backslash \mathcal{A}) \cap B(a, r))=0\right\},
$$

where for a subset $\mathcal{A} \subset \Omega$ the notation $\operatorname{Tan}(\mathcal{A}, a)$ stands for the cone

$$
\begin{gathered}
\operatorname{Tan}(\mathcal{A}, a)=\left\{v \in \mathbb{R}^{3}: \forall \varepsilon>0 \exists x \in \mathcal{A}, r \in(0, R)\right. \\
\text { such that }|x-a|<\varepsilon \text { and }|r(x-a)-v|<\varepsilon\} .
\end{gathered}
$$

If $0<\theta(a)<\infty$ and $\operatorname{VarTan}(\mu, a)$ consists of the only element $v=2^{-1} \theta(a) \mathcal{H}^{1}\lfloor\mathcal{T}$ concentrated on a one-dimensional subspace $\mathcal{T} \subset \mathbb{R}^{3}$, then $\operatorname{Tan}(\mu, a)=\mathcal{T}$. The following rectifiability result, cf. [4] section 2.8 , is a straightforward consequence of the definitions.

Proposition 5.7 Suppose that $\mu$ is a non-negative Radon measure in $\Omega$ with $\theta(x) \in \mathbb{R}$ for each $x \in \Omega$, and Tan $(\mu, x)$ is a one-dimensional subspace of $\mathbb{R}^{3}$ for $\mu$-almost every $x \in \Omega_{\text {sing }}=\{x: 0<\theta(x)<\infty\}$. Then every compact subset of $\Omega_{\text {sing }}$ is $\left(\mathcal{H}^{1}, 1\right)$-rectifiable and

$$
\int_{\Omega_{\text {sing }}} f(x) d \mu(x)=\frac{1}{2} \int_{\Omega_{\text {sing }}} f(x) \theta(x) d \mathcal{H}^{1}(x) \text { for all } f \in C_{0}(\Omega) .
$$

The main result of this section is the following theorem.

Theorem 5.8 Under the assumptions of Theorem 1.1,

(i) every compact subset of $\Omega_{\text {sing }}$ is $\left(\mathcal{H}^{1}, 1\right)$ - rectifiable;

(ii) for $\mu$-almost every $x \in \Omega_{\text {sing }}$ there is $\mathbf{s}(x) \in \mathbb{S}^{2}$ such that Tan $(\mu, x)=\operatorname{span}\{\mathbf{s}(x)\}$;

(iii) the measure $\mathcal{E}$ has the representation

$$
\int_{\Omega_{\text {sing }}} \varphi(x): d \mathcal{E}(x)=\frac{1}{2} \int_{\Omega_{\text {sing }}} \varphi(x): \mathbf{s}(x) \otimes \mathbf{s}(x) \theta(x) d \mathcal{H}^{1}(x)
$$

for all $\varphi \in C_{0}(\Omega)^{9}$.

Proof. We start with the observation that the components of measure $\mathcal{E}$ are absolutely continuous with respect to $\mu$ which implies the representation

$$
\int_{\Omega_{\text {sing }}} \psi(x) d \mathcal{E}(x)=\int_{\Omega_{\text {sing }}} \psi(x) M(x) d \mu(x) \quad \text { for all } \psi \in C_{0}(\Omega)^{9} .
$$

Here the matrix-valued function $M \in L^{1}\left(\Omega_{\text {sing }}, \mu\right)^{9}$ is non-negative and $\operatorname{Tr} M(x)=1 \mu$ almost everywhere in $\Omega_{\text {sing }}$. Note also that

$$
\lim _{r \rightarrow 0} \frac{1}{r} \int_{B(a, r)}|M(a)-M(x)| d \mu(x)=\lim _{r \rightarrow 0} \frac{1}{\mu(B(a, r))} \int_{B(a, r)}|M(a)-M(x)| d \mu(x)=0
$$

$\mathrm{RR} \mathrm{n}^{\circ} 5481$ 
for $\mu$-almost every $a \in \Omega_{\text {sing }}$. Next, represent the concentration set in the form $\Omega_{\text {sing }}=$ $\bigcup_{k \geq 1} \Omega_{k}, \Omega_{k}=\{x: 1 / k<\theta(x)<k\}$. The estimates from the geometric measure theory imply that $1 /(2 k) \mathcal{H}^{1}(A) \leq \mu(A) \leq k \mathcal{H}^{1}(A)$ for any Borel set $A \subset \Omega_{k}$. Hence, $\mu(A)=0$ for every set $A$ of zero $\mathcal{H}^{1}$-measure, and the measure $\mu$ is absolutely continuous with respect to the one-dimensional Hausdorff measure. Next recall, cf. 26, that for any function $f \in L^{1}(\Omega)$, there is a set $E$ of zero $\mathcal{H}^{1}$-measure such that

$$
\lim _{r \rightarrow 0} \frac{1}{r} \int_{B(a, r)}|f(x)| d x=0
$$

everywhere in $\Omega$ possibly except of the set $E$. Since $\mu$ is absolutely continuous with respect to the Hausdorff $\mathcal{H}^{1}$, this relation holds true for $\mu$-almost every $a \in \Omega$. In particular, the equalities

$$
\begin{aligned}
& \lim _{r \rightarrow 0} \frac{1}{r} \int_{B(a, r)} \varrho|\mathbf{u}|^{2} d x=\lim _{r \rightarrow 0} \frac{1}{r} \int_{B(a, r)} \varrho d x= \\
& \lim _{r \rightarrow 0} \frac{1}{r} \int_{B(a, r)}|\Pi| d x=\lim _{r \rightarrow 0} \frac{1}{r} \int_{B(a, r)}|g| d x=0
\end{aligned}
$$

holds true for $\mu$-almost all $a \in \Omega$. Passing to the limit in equations (11) and using the equalities (41), (56) we conclude that for every $\varphi \in C_{0}^{1}(\Omega)^{3}$,

$$
\begin{gathered}
\int_{\Omega}(\nabla \varphi: \varrho \mathbf{u} \otimes \mathbf{u}+\operatorname{div} \varphi \varrho) d x+\int_{\Omega_{\text {sing }}} \nabla \varphi: M(x) d \mu(x)= \\
\int_{\Omega} \nabla \varphi: \Pi d x-\int_{\Omega} \varphi \cdot g d x .
\end{gathered}
$$

Now fix an arbitrary $a \in \Omega_{\text {sing }}$ satisfying (57), (58) and a vector-valued function $\varphi \in$ $C_{0}^{1}\left(\mathbb{R}^{3}\right)^{3}$. Substituting $\varphi\left(\varepsilon^{-1}(x-a)\right)$ in (59) we arrive at

$$
\begin{gathered}
\frac{1}{\varepsilon} \int_{\Omega_{\text {sing }}} \nabla \varphi\left(\frac{x-a}{\varepsilon}\right): M(a) d \mu(x)= \\
\frac{1}{\varepsilon} \int_{\Omega_{\text {sing }}} \nabla \varphi\left(\frac{x-a}{\varepsilon}\right):(M(a)-M(x)) d \mu(x)+ \\
\frac{1}{\varepsilon} \int_{\Omega} \nabla \varphi\left(\frac{x-a}{\varepsilon}\right):(\Pi-\varrho \mathbf{u} \otimes \mathbf{u}) d x- \\
\frac{1}{\varepsilon} \int_{\Omega} \operatorname{div} \varphi\left(\frac{x-a}{\varepsilon}\right) \varrho d x-\frac{1}{\varepsilon} \int_{\Omega} \varphi\left(\frac{x-a}{\varepsilon}\right) \cdot g d x .
\end{gathered}
$$


Note that, by (57) and (58), the right side of this equality tends to 0 as $\varepsilon \rightarrow 0$. Choose an element $v \in \operatorname{VarTan}(\mu, a)$ and the sequence $\varepsilon_{i}$ satisfying (54). Substituting $\varepsilon=\varepsilon_{i}$ into (60) and passing to the limit we obtain

$$
\int_{\mathbb{R}^{3}} \nabla \varphi(x): M(a) d v(x)=0 \text { for all } \varphi \in C_{0}^{1}\left(\mathbb{R}^{3}\right)^{3} .
$$

It follows from this that the matrix-valued measure $M(a) v$ satisfies all assumptions of Lemma 2.2 with $\Pi=0, g=0$, and $\Omega=\mathbb{R}^{3}$. Replacing $\mathcal{E}$ by $M(a) v$ in identity (22) and noting that $\operatorname{Tr} M(a)=1$ we obtain

$$
\frac{1}{r} \int_{B(0, r)} d v+\int_{r \leq|y| \leq R} \frac{1}{|y|}(1-\mathbf{n} \otimes \mathbf{n}: M(a)) d v+\zeta_{0}(0, r)=\frac{1}{R} \int_{B(0, R)} d v
$$

where $\zeta_{0} \geq 0$ and $\mathbf{n}=y /|y|$. Since $r^{-1} v(B(0, r))=R^{-1} v(B(0, R))$, we conclude from this that

$$
\int_{r \leq|y| \leq R} \frac{1}{|y|}(1-\mathbf{n} \otimes \mathbf{n}: M(a)) d v=0 \text { for all } r, R .
$$

Therefore, $|y|-y \otimes y: M(a)=0$ for $v$-almost every $y \in \mathbb{R}^{3}$. It is possible if and only if there exists $\mathbf{s}(a) \in \mathbb{S}^{2}$ such that $M(a)=\mathbf{s}(a) \otimes \mathbf{s}(a)$ and $v=2^{-1} \theta(a) \mathcal{H}^{1}\lfloor\operatorname{span}\{\mathbf{s}(a)\}$. Hence $\operatorname{Tan}(\mu, a)=\operatorname{span}\{\mathbf{s}(a)\}$ for $\mu$-almost every $a \in \Omega_{\text {sing }}$. From this we conclude that the measure $\mu$ satisfies the hypothesis of Proposition 5.7 Applying this proposition we obtain (55) which completes the proof of Theorem 5.8

We return to the proof of Theorem 1.1 It remains to note that the statement of Theorem 1.1 for $\gamma=1$ is an obvious consequence of Theorems 5.1 and 5.8 .

\section{Proof of Theorems 1.2 and 1.3}

Proof of Theorem 1.2 In order to avoid repetitions we give only the proof for $\gamma>1$. Fix an arbitrary $\varepsilon>0$ and consider the family of boundary-value problems, depending on parameter $t \in[0,1]$,

$$
\begin{gathered}
\left(\alpha \varrho+\sqrt{\varepsilon} \varrho^{5}\right) \mathbf{u}+\operatorname{div}(\mathbf{u} \otimes(t \varrho \mathbf{u}-\varepsilon \nabla \varrho))+t \nabla p(\varrho)=t \varrho \mathbf{F}+\operatorname{div} \Sigma(\mathbf{u}) \text { in } \Omega \\
\alpha \varrho+\sqrt{\varepsilon} \varrho^{5}+t \operatorname{div}(\varrho \mathbf{u})-\varepsilon \Delta \varrho=t f \text { in } \Omega, \quad u=0, \partial_{n} \varrho=0 \text { on } \partial \Omega .
\end{gathered}
$$

We begin with proving a priori estimates for solution to problem (63). Multiplying the both sides of equations (63a) and (63b) by $\frac{\gamma-1}{\gamma} \mathbf{u}$ and $\varrho^{\gamma-1}$, respectively, integrating over $\Omega$, and 
combining the obtained results we arrive at the identity

$$
\begin{gathered}
\frac{\gamma-1}{\gamma} \int_{\Omega}\left(|\nabla \mathbf{u}|^{2}+(1+\nu)|\operatorname{div} \mathbf{u}|^{2}+\frac{\varrho\left|\mathbf{u}^{2}\right|}{2}\left(\alpha+\sqrt{\varepsilon} \varrho^{4}\right)\right) d x+ \\
\int_{\Omega}\left(\alpha \varrho^{\gamma}+\sqrt{\varepsilon} \varrho^{4+\gamma}+\varepsilon(\gamma-1) \varrho^{\gamma-2}|\nabla \varrho|^{2}\right) d x= \\
\int_{\Omega}\left(t \frac{\gamma-1}{\gamma} \varrho \mathbf{F u}+f\left(\varrho^{\gamma-1}-\frac{|\mathbf{u}|^{2}}{2}\right)\right) d x,
\end{gathered}
$$

which holds true for every smooth solution $(\varrho, \mathbf{u})$, with a positive density $\varrho$, to problem (63). Noting that for $\nu>-2$,

$$
\int_{\Omega}\left(|\nabla \mathbf{u}|^{2}+(1+\nu)|\operatorname{div} \mathbf{u}|^{2}\right) d x \geq c(\nu) \int_{\Omega}|\nabla \mathbf{u}|^{2} d x, \text { where } c(\nu)>0,
$$

we obtain the estimates

$$
\begin{gathered}
\|\varrho\|_{L^{\gamma}(\Omega)}+\|\mathbf{u}\|_{H^{1,2}(\Omega)}+\left\|\varrho|\mathbf{u}|^{2}\right\|_{L^{1}(\Omega)} \leq c \\
\varepsilon^{1 / 2}\left\|\varrho^{4+\gamma}\right\|_{L^{1}(\Omega)}+\varepsilon^{1 / 2}\left\|\varrho^{5}|\mathbf{u}|^{2}\right\|_{L^{1}(\Omega)}+\varepsilon\left\|(1+\varrho)^{2-\gamma}|\nabla \varrho|^{2}\right\|_{L^{1}(\Omega)} \leq c
\end{gathered}
$$

with a constant $c$ independent of $t$ and $\varepsilon$. Next, multiplying the both sides of (63b) by $\sqrt{\varepsilon} \varrho^{m-1}, 1 \leq m \leq 4$, and integrating the result over $\Omega$ we obtain

$$
\begin{gathered}
\int_{\Omega}\left(\varepsilon^{3 / 2}(m-1) \varrho^{m-2}|\nabla \varrho|^{2}+\varepsilon^{1 / 2} \alpha \varrho^{m}+\varepsilon \varrho^{4+m}\right) d x= \\
\sqrt{\varepsilon} \int_{\Omega}\left(\varrho^{m-1} f-t \frac{m-1}{m} \varrho^{m} \operatorname{div} \mathbf{u}\right) d x .
\end{gathered}
$$

Noting that

$$
\left|\int_{\Omega} \varrho^{m-1} f d x\right|+\left|\int_{\Omega} \varrho^{m} \operatorname{div} \mathbf{u} d x\right| \leq c_{1}\left(\int_{\Omega} \varrho^{2 m}\right)^{1 / 2} \leq c_{2}\left(\int_{\Omega} \varrho^{4+m}\right)^{1 / 2}+c_{3},
$$

we arrive at the inequality

$$
\int_{\Omega}\left(\varepsilon^{3 / 2}(m-1) \varrho^{m-2}|\nabla \varrho|^{2}+\varepsilon^{1 / 2} \alpha \varrho^{m}+\varepsilon \varrho^{4+m}\right) d x \leq c_{4} \sqrt{\varepsilon}\left(\int_{\Omega} \varrho^{4+m}\right)^{1 / 2}+c_{5},
$$

which with $m=2$ and $m=4$ gives the estimates

$$
\varepsilon^{3 / 4}\|\nabla \varrho\|_{L^{2}(\Omega)}+\varepsilon^{1 / 8}\|\varrho\|_{L^{8}(\Omega)} \leq c .
$$

INRIA 
Next note that (64)-(66) imply the estimates

$$
\begin{gathered}
\|\varrho \nabla \mathbf{u}\|_{L^{8 / 5}(\Omega)} \leq\|\nabla \mathbf{u}\|_{L^{2}(\Omega)}\|\varrho\|_{L^{8}(\Omega)} \leq c \varepsilon^{-1 / 8} \\
\|\mid \mathbf{u}\| \nabla \varrho\|\|_{L^{3 / 2}(\Omega)} \leq\|\mathbf{u}\|_{L^{6}(\Omega)}\|\nabla \varrho\|_{L^{2}(\Omega)} \leq c \varepsilon^{-3 / 4} \\
\|\varrho \mathbf{u}\|_{L^{24 / 7}(\Omega)} \leq\|\mathbf{u}\|_{L^{6}(\Omega)}\|\varrho\|_{L^{8}(\Omega)} \leq c \varepsilon^{-1 / 8} \\
\left\|\varrho^{5} \mid\right\|_{L^{8 / 5}(\Omega)} \leq\|\varrho\|_{L^{8}(\Omega)}^{5} \leq c \varepsilon^{-5 / 8}
\end{gathered}
$$

It is easy to see that a solution to (63a) has the representation $\varrho=\varrho_{1}+\varrho_{2}$ in which $\varrho_{i}$ satisfy the equations

$$
(\varepsilon \Delta-\alpha) \varrho_{1}=t \operatorname{div}(\varrho \mathbf{u}), \quad(\varepsilon \Delta-\alpha) \varrho_{2}=\sqrt{\varepsilon} \varrho^{5},
$$

and the homogeneous Neumann boundary conditions. From this, in view of the embeddings $H^{1,24 / 7}(\Omega) \hookrightarrow C(\Omega), H^{2,8 / 5}(\Omega) \hookrightarrow C(\Omega)$, and inequalities 67c), 67d , we conclude that $\varrho \leq c_{\varepsilon}$, where a constant $c_{\varepsilon}$ does not depend on $t$. Since $\varrho$ is bounded from above, equation (63a) can be rewritten in the form

$$
\Delta \mathbf{u}+(1+\nu) \nabla \operatorname{div} \mathbf{u}=\operatorname{div} G_{2}+G_{1} \text { in } \Omega,
$$

with the terms in the right side satisfying the inequalities $\left|G_{i}\right| \leq c_{\varepsilon}\left(1+|\mathbf{u}|^{i}\right), i=1,2$. Embedding $H^{1,3}(\Omega) \hookrightarrow L^{r}(\Omega), r>1$, inequality (64) and a priori estimates for elliptic equations yield $\|\mathbf{u}\|_{L^{r}(\Omega)} \leq c_{\varepsilon}(r)$. Arguing as before we conclude that $\|\mathbf{u}\|_{H^{1, r}(\Omega)} \leq c_{\varepsilon}(r)$ for every $r>1$ and hence $|\mathbf{u}| \leq c_{\varepsilon}$. Therefore, $\varrho$ and $|\mathbf{u}|$ are bounded by a constant independent of $t$. From this and the results from the theory of weakly nonlinear elliptic equations, see Theorem 13.1 in [3], we conclude that the inequality

$$
\|(\varrho, \mathbf{u})\|_{C^{2+\beta}(\Omega)}<C^{*}\left(\varepsilon, \Omega,\|(f, \mathbf{F})\|_{C^{\beta}(\Omega)}, \alpha, \nu\right)
$$

holds for every solution $(\varrho, \mathbf{u}) \in C^{1+\beta}(\Omega), \varrho>0$, to problem (63).

To tackle the existence question we need to reformulate problem (63) as a nonlinear operator equation in the form $(\varrho, \mathbf{u})=\Phi_{t}(\varrho, \mathbf{u})$. Introduce the mapping $\Phi_{t}:(\varrho, \hat{\mathbf{u}}) \mapsto(\varrho, \mathbf{u})$ defined as a solution to the boundary-value problem

$$
\begin{gathered}
\operatorname{div} \Sigma(\mathbf{u})=\left(\alpha \hat{\varrho}+\sqrt{\varepsilon} \varrho^{5}\right) \hat{\mathbf{u}}+\operatorname{div}((t \varrho \hat{\mathbf{u}}-\varepsilon \nabla \hat{\varrho}) \otimes \hat{\mathbf{u}})+t \nabla p(\varrho)-t \varrho \hat{F} \\
\varepsilon \Delta \varrho-\alpha \varrho=\sqrt{\varepsilon} \varrho^{4} \varrho+t \operatorname{div}(\varrho \hat{\mathbf{u}})-f \text { in } \Omega, \quad u=0, \quad \partial_{n} \varrho=0 \text { on } \partial \Omega .
\end{gathered}
$$

Obviously, the mapping $(\hat{\varrho}, \hat{\mathbf{u}}, t) \mapsto(\varrho, \mathbf{u})$ denoted by $\Phi: C^{1+\beta}(\Omega)^{4} \times[0,1] \mapsto C^{2+\beta}(\Omega)^{4}$ is continuous. The remaining part of the proof relies on the following lemma, but first we recall an abstract result useful for our purposes.

The proof of Lemma 6.2 is based on the following result from the theory of positive operators, cf. [18, ch.7.3.10. Let $E_{1}, E_{2}$ be Banach spaces with the cones $\mathbb{K}_{i} \subset E_{i}, i=1,2$. A bounded operator $A: E_{2} \mapsto E_{1}$ is said to be positive if $A \mathbb{K}_{2} \subset \mathbb{K}_{1}$. 
Proposition 6.1 Suppose that bounded operators $B_{1}, B_{2}: E_{1} \mapsto E_{2}$ satisfy the following conditions:

1. The operator $B_{1}$ has the bounded, positive inverse.

2. The operator $B_{1}^{-1}\left(B_{1}-B_{2}\right)$ is compact.

3. There is an element $\varrho_{0} \in$ Int $\mathbb{K}_{1}$ and a positive constant $\varkappa$ such that $B_{2} \varrho_{0} \geq \varkappa B_{1} \varrho_{0}$.

4. $\left(B_{1}-B_{2}\right) u \in \mathbb{K}_{2}$ for all $u \in \mathbb{K}_{1}$, in other words, $B_{1} \geq B_{2}$.

Then the operator $B_{2}$ has a positive inverse.

The following lemma is an application of the abstract result.

Lemma 6.2 Let $\Omega \subset \mathbb{R}^{3}$ be a bounded domain with the boundary $\partial \Omega \in C^{2+\beta}$, vector field $\mathbf{u} \in C^{1+\beta}(\Omega)^{3}$ vanishes on $\partial \Omega$, and a function $b \in C^{\beta}(\Omega)$ is strictly positive in the closure $\mathrm{cl} \Omega$. Then for any non-negative $f \in C^{\beta}(\Omega)$ the problem

$$
-\varepsilon \Delta \varrho+\operatorname{div}(\varrho \mathbf{u})+b \varrho=f \quad \text { in } \Omega, \partial_{n} \varrho=0 \text { on } \partial \Omega,
$$

has a unique strictly positive solution $\varrho \in C^{2+\beta}(\Omega)$

Proof. Now denote by $E_{1}$ the closed subspace of $C^{2+\beta}(\Omega)$ which consists of all functions satisfying the homogenous boundary Neumann condition on $\partial \Omega$, and set $E_{2}=C^{\beta}(\Omega)$. Let $\mathbb{K}_{i} \subset E_{i}, i=1,2$, be the cones of non-negative functions. Let $L: E_{1} \mapsto E_{2}$ be a linear operator defined by $L \varrho \equiv-\varepsilon \Delta \varrho+\operatorname{div}(\varrho \mathbf{u})$. Hence our task is to prove that there exists the bounded positive operator $(L+b)^{-1}: E_{2} \mapsto E_{1}$. We start with proving that the equation $L \varrho_{0}=0$ has a strictly positive solution $\varrho_{0} \in E_{1}$. It follows from the general theory of boundary-value problems for the second order elliptic differential equations [16 that for fixed $k>\sup (|\operatorname{div} \mathbf{u}(x)|+b(x))$ and all $f \in E_{2}$, the Neumann problem

$$
(L+k) \varrho=f \in E_{2}, \quad \varrho \in E_{1}
$$

has the unique non-negative solution. By the maximum principle $\varrho$ is non-negative if $f$ is non-negative. Hence the compact positive operator is defined by $A=(L+k)^{-1}: E_{2} \mapsto E_{2}$. We can apply the maximum principle ([16, Theorem 9.6) for (71), to obtain that $\varrho$ does not attain the non-positive minimum in $\Omega$. Hence a solution to problem (71) is strictly positive inside $\Omega$ for all $f \in \mathbb{K}_{2} \backslash\{0\}$. Moreover, the solution is strictly positive in the closure of $\Omega$. In order to prove the last statement suppose, on the contrary to our claim, that $A f\left(x_{0}\right)=0$ at some point $x_{0} \in \partial \Omega$. Observe that the function $\varrho=A f \in E_{1}$ is positive inside $\Omega$ and satisfies the inequality $(L+k) \varrho \geq 0$ in $\Omega$. Furthermore, $\varrho$ takes the zero value for the minimum at $x_{0}$. By Lemma 3.4 in [16] the interior normal derivative of $\varrho$ at point $x_{0}$ is strictly positive, which is in contradiction with the homogeneous Neumann boundary condition. 
Set $\varrho_{1}=A 1$, where 1 is the characteristic function of $\Omega$. Since $A f$ continuous and strictly positive, for every $f \in \mathbb{K}_{2} \backslash\{0\}$ there exist positive constants $\alpha, \beta$ depending on $f$ such that $\alpha \varrho_{1} \leq A f \leq \beta \varrho_{1}$. Hence $A: E_{2} \mapsto E_{2}$ is a compact $\varrho_{1}$-positive operator, [17. Classical results from theory of positive operators, see Theorems 2.8, 2.10 and 2.13 from [17] for example, imply that $A$ has a positive simple eigenvalue $\lambda_{0}$ such that the corresponding eigenfunction is strictly positive and $\lambda_{0}>|\lambda|$ for any eigenvalue $\lambda \neq \lambda_{0}$.

Our next task is to show that $\lambda_{0}=(k)^{-1}$. We begin with observation that the operator equation $(k)^{-1} \varrho-A \varrho=\mathbf{1}$ is equivalent to the boundary-value problem $L \varrho=k, \varrho \in E_{1}$, which has no solutions. Therefore, by the Fredholm alternative, $k^{-1}$ is an eigenvalue of the operator $A$. Let us prove that $k^{-1}$ is the maximal eigenvalue. If this assertion is false, then there exists the positive eigenvalue $\lambda_{0}>k^{-1}$. By the definition of $A$, the eigenfunction $\varrho_{0} \in E_{1}$ satisfying the equation $\lambda_{0} \varrho_{0}-A \varrho_{0}=0$ is a solution to the boundary-value problem $(L+\tau) \varrho_{0}=0, \varrho_{0} \in E_{1}$, where $\tau=k-\lambda_{0}^{-1}>0$. Let us consider the parabolic boundary-value problem

$$
\begin{gathered}
\frac{\partial v}{\partial t}-\varepsilon \Delta v+\operatorname{div}(v \mathbf{u})=0 \text { in } \Omega \times(0, \infty), \quad \nabla v \cdot \mathbf{n}=0 \text { on } \partial \Omega \times(0, \infty), \\
v(x, 0)=v_{0}(x) \text { in } \Omega .
\end{gathered}
$$

For any $v_{0} \in C^{\beta}(\Omega)$ this problem has the unique smooth solution, which is positive if $v_{0}$ is non-negative. Introduce the operator $V(t): v_{0}(\cdot) \mapsto v(\cdot, t)$. Obviously $V(t)$ preserves the order and the charge i.e., $V(t) v_{0}^{\prime} \geq V(t) v_{0}$ for any $v_{0}^{\prime} \geq v_{0}$ and $\int_{\Omega} V(t) v_{0}(x) d x=\int_{\Omega} v_{0} d x$. Since for every $u, v \in C^{\beta}(\Omega)$ the function $\max \{u, v\} \in C^{\beta}(\Omega)$ we can apply the CrandallTartar Theorem [7 which implies that $V(t)$ is a non-expansive operator in the metric of $L^{1}(\Omega)$. In particular, we have $\left\|V(t) \varrho_{0}\right\|_{L^{1}(\Omega)}=\left\|\varrho_{0}\right\|_{L^{1}(\Omega)}$. On the other hand, equation $(L+\tau) \rho_{0}=0$ implies that $V(t) \varrho_{0}=e^{\tau t} \varrho_{0}$. Hence $\tau=0$ which gives $\lambda_{0}=k^{-1}$ and the assertion follows. Recall that $\varrho_{0}$ satisfies the equation $L \varrho_{0}=0$.

Therefore, the operators $B_{1}=(L+k), B_{2}=(L+b): E_{2} \mapsto E_{1}$ are continuous and the inverse $(L+k)^{-1}=A: E_{1} \mapsto E_{2}$ is positive. Moreover, $L+k \geq L+b$ and $(L+b) \varrho_{0}=$ $b \varrho_{0} \geq \varkappa k \varrho_{0}=\varkappa(L+k) \varrho_{0}$ for some positive $\varkappa$. Obviously $\varrho_{0}>0$ is the interior point of the cones $\mathbb{K}_{1}$ and $\mathbb{K}_{2}$. From this we conclude that the operator $L+b$ has the bounded positive inverse, which completes the proof.

Let us turn back to the proof of Theorem 1.2 Denote by $\mathcal{J}$ a subset of $C^{1+\beta}(\Omega)^{4}$ defined by the inequalities $\left\{(\varrho, \mathbf{u}): \varrho \geq 0,\|(\varrho, \mathbf{u})\|_{C^{1+\beta}(\Omega)} \leq C^{*}\right\}$. It follows from Lemma 6.2 that every fixed point $(\varrho, \mathbf{u})$ of $\Phi_{t}$ satisfies inequality (68). Moreover, $\varrho \in \mathcal{J}$ is strictly positive. Hence there are no fixed points of $\Phi_{t}$ at $\partial \mathcal{J}$ for all $t \in[0,1]$. On the other hand, the mapping $\Phi_{0}$ has the unique fixed point inside $\mathcal{J}$. By the Leray-Schauder fixed point Theorem, problem (63) has a solution $(\varrho, \mathbf{u}) \in$ int $\mathcal{J}$. It remains to note that estimates (15) follows from (64)-67d and the proof of Theorem [1.2] is completed.

Proof of Theorem 1.3 The proof is based on the following lemmas. 
Lemma 6.3 Let $\left(\mathbf{u}_{\varepsilon}, \varrho_{\varepsilon}\right)$ be a sequence of solutions to problem (14). Then there is a subsequence, still denoted by $\left(\mathbf{u}_{\varepsilon}, \varrho_{\varepsilon}\right)$, such that

$\mathbf{u}_{\varepsilon} \rightarrow \mathbf{u}$ weakly in $H_{0}^{1,2}(\Omega), \varrho_{\varepsilon} \rightarrow \varrho$ weakly in $L^{\gamma}(\Omega), \varrho_{\varepsilon} \mathbf{u}_{\varepsilon} \rightarrow \varrho \mathbf{u}$ weakly in $L^{1}(\Omega)$,

$$
\sqrt{\varepsilon}\left\|\varrho_{\varepsilon}^{5}\right\|_{L^{1}(\Omega)}+\sqrt{\varepsilon}\left\|\varrho_{\varepsilon}^{5} \mathbf{u}_{\varepsilon}\right\|_{L^{1}(\Omega)}+\varepsilon\left\|\left|\mathbf{u}_{\varepsilon}\right| \nabla \varrho_{\varepsilon}\right\|_{L^{3 / 2}(\Omega)} \rightarrow 0 \text { as } \varepsilon \rightarrow 0 .
$$

Proof. The first two convergences in (172) obviously follows from Theorem 1.2 In order to prove the third convergence note that by the Egoroff's theorem for every $\eta>0$ there exists $E \subset \Omega$ such that $\mathbf{u}_{\varepsilon} \rightarrow \mathbf{u}$ uniformly on $E$, and meas $(\Omega \backslash E)<\eta$ which yields

$$
\begin{gathered}
\limsup _{\varepsilon \rightarrow 0}\left|\int_{\Omega} h\left(\varrho_{\varepsilon} \mathbf{u}_{\varepsilon}-\varrho \mathbf{u}\right) d x\right| \leq 2\|h\|_{L^{\infty}(\Omega)} \limsup _{\varepsilon \rightarrow 0} \int_{E} \varrho_{\varepsilon}\left|\mathbf{u}_{\varepsilon}\right| d x \leq \\
c \limsup _{\varepsilon \rightarrow 0}\left(\int_{E} \varrho_{\varepsilon} d x\right)^{1 / 2}\left(\int_{E} \varrho_{\varepsilon}\left|\mathbf{u}_{\varepsilon}\right|^{2} d x\right)^{1 / 2} \leq c \limsup _{\varepsilon \rightarrow 0}\left(\int_{E} \varrho_{\varepsilon} d x\right)^{1 / 2} \rightarrow 0 \text { as } \eta \rightarrow 0 .
\end{gathered}
$$

Since, by (15b) and (15e), the sequence $\sqrt{\varepsilon} \varrho_{\varepsilon}^{5} \ln \left(1+\varrho_{\varepsilon}\right)$ is bounded in $L^{1}(\Omega)$, we have

$$
\begin{gathered}
\limsup _{\varepsilon \rightarrow 0} \sqrt{\varepsilon} \int_{\Omega} \varrho_{\varepsilon}^{5} d x \leq \limsup _{\varepsilon \rightarrow 0} \sqrt{\varepsilon} \int_{\varrho_{\varepsilon}>N} \varrho_{\varepsilon}^{5} d x \leq \\
\ln (1+N)^{-1} \limsup _{\varepsilon \rightarrow 0} \sqrt{\varepsilon} \int_{\varrho_{\varepsilon}>N} \varrho_{\varepsilon}^{5} \ln \left(1+\varrho_{\varepsilon}\right) d x \leq c \ln (1+N)^{-1} \rightarrow 0 \text { as } N \rightarrow \infty
\end{gathered}
$$

which implies the convergence of the first term in (73). Noting that

$$
\sqrt{\varepsilon}\left\|\varrho_{\varepsilon}^{5} \mathbf{u}_{\varepsilon}\right\|_{L^{1}(\Omega)} \leq N \sqrt{\varepsilon}\left\|\varrho_{\varepsilon}^{5}\right\|_{L^{1}(\Omega)}+N^{-1} \sqrt{\varepsilon}\left\|\varrho_{\varepsilon}^{5}\left|\mathbf{u}_{\varepsilon}\right|^{2}\right\|_{L^{1}(\Omega)} \leq N \sqrt{\varepsilon}\left\|\varrho_{\varepsilon}^{5}\right\|_{L^{1}(\Omega)}+c N^{-1}
$$

we obtain $\limsup _{\varepsilon \rightarrow 0} \sqrt{\varepsilon}\left\|\varrho_{\varepsilon}^{5} \mathbf{u}_{\varepsilon}\right\|_{L^{1}(\Omega)} \leq c N^{-1}$, which gives the convergence of the second term in (73). It remains to note that, by (15d),

$$
\varepsilon\left\|\left|\mathbf{u}_{\varepsilon}\right| \nabla \varrho_{\varepsilon}\right\|_{L^{3 / 2}(\Omega)} \leq \varepsilon\left\|\nabla \varrho_{\varepsilon}\right\|_{L^{2}(\Omega)}\left\|\mathbf{u}_{\varepsilon}\right\|_{L^{6}(\Omega)} \leq c \varepsilon^{1 / 4},
$$

and the lemma follows.

Lemma 6.4 If $\gamma=1$, then $\varrho_{\varepsilon} \mathbf{u}_{\varepsilon} \otimes \mathbf{u}_{\varepsilon} \rightarrow \varrho \mathbf{u} \otimes \mathbf{u}+\mathcal{S}$ in $\mathcal{D}^{\prime}(\Omega)$, where $\mathcal{S}$ meets all requirements of Theorem [1.1. If $\gamma>1$ then for any $\Omega^{\prime} \Subset \Omega$,

$$
\varrho_{\varepsilon} \rightarrow \varrho \text { weakly in } L^{\gamma(1+\kappa)}\left(\Omega^{\prime}\right), \quad \varrho_{\varepsilon} \mathbf{u}_{\varepsilon} \otimes \mathbf{u}_{\varepsilon} \rightarrow \varrho \mathbf{u} \otimes \mathbf{u} \text { weakly in } L^{(1+\kappa)}\left(\Omega^{\prime}\right) .
$$

Proof. We start with he observation that equation (14a) can be written in the form (11) with the terms in the right side defined by

$$
\Pi_{\varepsilon}^{(1)}=\Sigma\left(\mathbf{u}_{\varepsilon}\right), \quad \Pi_{\varepsilon}^{(2)}=-\varepsilon \nabla \varrho_{\varepsilon} \otimes \mathbf{u}_{\varepsilon}, \quad g_{\varepsilon}=\varrho_{\varepsilon} \mathbf{F}-\varrho_{\varepsilon} \mathbf{u}_{\varepsilon}-\sqrt{\varepsilon} \varrho_{\varepsilon}^{5} \mathbf{u}_{\varepsilon} .
$$


Hence the lemma will be proved if we show that the functions satisfy all assumptions of Theorem 1.1 Since the sequence $\Pi_{\varepsilon}^{(1)}$ is bounded in $L^{2}(\Omega)^{9}$, it follows from (72) and (73) that it suffices to show that $\Pi_{\varepsilon}^{(2)}$ satisfy (9). To this end note that formulae (10) and (74) yield the representation

$$
\mathfrak{S} \Pi_{\varepsilon}^{(2)}(x, r, R)=\int_{r}^{R} \frac{d t}{t} \int_{|x-y|=t} \delta_{i} \varrho_{\varepsilon} \mathbf{u}_{\varepsilon, i} d S,
$$

where $\delta_{i}=\partial_{i}-\mathbf{n}_{i} \mathbf{n}_{k} \partial_{k}$ denotes the tangential derivatives on the sphere $\left\{y \in \mathbb{R}^{3}:|x-y|=t\right\}$ for a fixed $x$. Integrating by parts over this sphere we obtain the identity

$$
\mathfrak{S} \Pi_{\varepsilon}^{(2)}(x, r, R)=-\int_{r}^{R} \frac{d t}{t} \int_{|x-y|=t} \varrho_{\varepsilon} \delta_{i} \mathbf{u}_{\varepsilon, i} d S-\int_{r}^{R} \frac{d t}{t^{2}} \int_{|x-y|=t} \varrho_{\varepsilon} \mathbf{u}_{\varepsilon} \cdot \mathbf{n} d S,
$$

which along with (15c) implies the estimate

$$
\begin{gathered}
\left|\mathfrak{S} \Pi_{\varepsilon}^{(2)}(x, r, R)\right| \leq \varepsilon \int_{B(x, R)}\left(\frac{\left|\varrho_{\varepsilon} \nabla \mathbf{u}_{\varepsilon}\right|}{|y|}+\frac{\left|\varrho_{\varepsilon} \mathbf{u}_{\varepsilon}\right|}{|y|^{2}}\right) d y \leq \\
c \varepsilon\left\|\varrho_{\varepsilon} \nabla \mathbf{u}_{\varepsilon}\right\|_{L^{8 / 5}(\Omega)}+c \varepsilon\left\|\varrho_{\varepsilon} \mathbf{u}_{\varepsilon}\right\|_{L^{27 / 4}(\Omega)} \leq c \varepsilon^{7 / 8} \rightarrow 0 \text { as } \varepsilon \rightarrow 0 .
\end{gathered}
$$

Hence $\Pi_{\varepsilon}^{(i)}$ and $g_{\varepsilon}$ satisfy all conditions of Theorem 1.1 which completes the proof.

It follows from Lemma 6.4 that for every $\Omega^{\prime} \Subset \Omega$ the sequence $p_{\varepsilon}$ converges weakly in $L^{1+\kappa}\left(\Omega^{\prime}\right)$ to some function $\bar{p} \in L^{1}(\Omega) \cap L_{l o c}^{1+\kappa}(\Omega)$. The following theorem plays an important role in the proof of Theorem 1.3

Theorem 6.5 Let $\gamma>1$. Then $\bar{p}=p(\varrho)$.

Proof. Sections 78 are devoted to the proof.

It remains to note that the the statement of Theorem 1.3 is an obvious consequence of Lemmas 6.3, 6.4 and Theorem 6.5

\section{Young measures, viscosity flux, and oscillations}

Young measures and distribution functions In this paragraph we give the representations of weak limits of approximate solutions via the Young measures. Let us consider the sequence of solutions to problem (14). It follows from Lemma 6.4 that there exists a subsequence, still denoted by $\left(\varrho_{\varepsilon}, \mathbf{u}_{\varepsilon}\right)$, which enjoys the following property. Let positive $\gamma$ and $\kappa$ be given. For any continuous function $G: \Omega \times \mathbb{R}_{\lambda} \mapsto \mathbb{R}$ satisfying the conditions

$$
(1+|\lambda|)^{-\gamma(1+\kappa)} \sup _{x \in \Omega}|G(x, \lambda)| \rightarrow 0 \text { as }|\lambda| \rightarrow \infty
$$


and $\Omega^{\prime} \Subset \Omega$, the sequence $G\left(x, \varrho_{\varepsilon}\right)$ converges weakly in $L^{1}\left(\Omega^{\prime}\right)$ to some function $\bar{G} \in L_{l o c}^{1}(\Omega)$. Moreover, for any continuous bounded function $\varphi: \mathbb{R} \mapsto \mathbb{R}$, the functions $\varphi\left(\varrho_{\varepsilon}\right) \operatorname{div} \mathbf{u}_{\varepsilon}$ converge weakly in $L^{2}(\Omega)$ to some function $\overline{\varphi \operatorname{div} \mathbf{u}}$. The Ball's version [5] of the fundamental theorem on Young measures implies the following result.

Lemma 7.1 There exists a weakly measurable family of probability measures $\sigma_{x} \in C_{0}(\mathbb{R})^{*}$ with spt $_{x} \subset[0, \infty)$ such that the equality

$$
\bar{G}=\int_{[0, \infty)} G(x, \lambda) d \sigma_{x}(\lambda)
$$

holds for any function $G$ satisfying condition (75). Moreover, the function

$$
x \mapsto \int_{[0, \infty)} \lambda^{\gamma(1+\kappa)} d \sigma_{x}(\lambda)
$$

belongs to $L_{l o c}^{1}(\Omega)$. If the function $G$ satisfy more restrictive condition $(1+|\lambda|)^{-\gamma} \sup _{x \in \Omega}|G(x, \lambda)| \rightarrow$ 0 for $|\lambda| \rightarrow \infty$, then $G\left(x, \varrho_{\varepsilon}\right) \rightarrow \bar{G}$ weakly in $L^{1}(\Omega)$. In particular, the functions $\bar{p} \in L^{1}(\Omega)$ and $\varrho \in L^{\gamma}(\Omega)$ are given by

$$
\bar{p}(x)=\int_{[0, \infty)} \lambda^{\gamma} d \sigma_{x}(\lambda), \quad \varrho(x)=\int_{[0, \infty)} \lambda d \sigma_{x}(\lambda) .
$$

By technical reasons it is convenient to replace the measure $\sigma_{x}$ with its distribution function $\Gamma(x, \lambda):=\sigma_{x}(-\infty, \lambda]$ such that the measure $\sigma_{x}$ is the Stieltjes measure $d_{\lambda} \Gamma(x, \lambda)$. The distribution function is measurable with respect to $(x, \lambda) \in \Omega \times \mathbb{R}_{\lambda}$, monotone and continuous from the right in $\lambda$,

$$
\Gamma(x, \lambda)=0 \text { for } \lambda<0, \quad \Gamma(x, \lambda) \nearrow 1 \text { as } \lambda \nearrow \infty .
$$

Recalling that $\sigma_{x}$ is the Stieltjes measure associated with $\Gamma(x, \cdot)$ we get the following formulae

$$
\bar{p}(x)=\gamma \int_{[0, \infty)} \lambda^{\gamma-1}(1-\Gamma(x, \lambda)) d \lambda, \quad \varrho(x)=\int_{[0, \infty)}(1-\Gamma(x, \lambda)) d \lambda .
$$

Remark 7.2 Relations (79) imply that equality $\Gamma(1-\Gamma)=0$ a.e. in $\Omega \times \mathbb{R}_{\lambda}$ which yields $\bar{p}=p(\varrho)$.

In the rest of this paragraph we consider the family of Radon measures $m_{\varepsilon}$ on $\Omega \times \mathbb{R}_{\lambda}$ defined by

$$
\int_{\Omega \times \mathbb{R}_{\lambda}} \Phi(x, \lambda) d m_{\varepsilon}(x, \lambda):=\varepsilon \int_{\Omega} \Phi\left(x, \varrho_{\varepsilon}(x)\right)\left|\nabla \varrho_{\varepsilon}(x)\right|^{2} d x
$$

INRIA 
for each $\Phi \in C_{0}\left(\Omega \times \mathbb{R}_{\lambda}\right)$. It follows from Theorem 1.2 that there exist a subsequence, still denoted by $\left\{\varrho_{\varepsilon}\right\}$, and a Radon measure $m$ on $\Omega \times \mathbb{R}_{\lambda}$ such that

$$
\int_{\Omega \times \mathbb{R}_{\lambda}} \Phi(x, \lambda) d m_{\varepsilon}(x, \lambda) \rightarrow \int_{\Omega \times \mathbb{R}_{\lambda}} \Phi(x, \lambda) d m(x, \lambda) \text { for all } \Phi \in C_{0}\left(\Omega \times \mathbb{R}_{\lambda}\right) .
$$

Obviously $m(\Omega \times(-\infty, 0))=0$. Moreover, estimates (15d) from Theorem 1.2 yield

$$
\int_{\Omega \times \mathbb{R}_{\lambda}}(1+|\lambda|)^{-1} d m(x, \lambda)<\infty .
$$

The effective viscosity flux Following [19] we introduce the quantity $V(\varrho, \mathbf{u})=p(\varrho)-$ $(2+\nu) \operatorname{div} \mathbf{u}$ called the effective viscous flux. As it was shown in 19, 12, 14, the effective viscous flux enjoys many remarkable properties. The most important is the multiplicative relation $\overline{\varphi(\varrho) V}=\overline{\varphi(\varrho)} \bar{V}$ which was proved in [19] for all $\gamma>3 / 2$. The simple proof of this result, based on the new version of compensated compactness principle, was given in papers [12, 14. In our case, by Lemma 6.4 the critical estimate $\left\|\varrho_{\varepsilon}\left|\mathbf{u}_{\varepsilon}\right|^{2}\right\|_{L^{(1+\kappa)}\left(\Omega^{\prime}\right)} \leq c\left(\Omega^{\prime}\right)$ holds for every $\Omega^{\prime} \Subset \Omega$, which leads to the following local version of the compensated compactness result from $[14$.

Let us consider a function $\Phi \in C(\Omega \times \mathbb{R})$ such that

$$
\Phi(\cdot, \lambda) \in C_{0}(\Omega) \text { for all } \lambda \in \mathbb{R}^{+}, \quad \Phi(\cdot, \lambda)=\Phi_{\infty}(\cdot) \in C_{0}(\Omega) \text { for all } \lambda>N>0 .
$$

Lemma 7.3 Let $\left(\mathbf{u}_{\varepsilon}, \varrho_{\varepsilon}\right)$ be a sequence of solutions to problem (14) satisfying the hypotheses of Theorem 1.2] Then for $\Phi \in C(\Omega \times \mathbb{R})$ satisfying (83),

$$
\int_{\Omega} \overline{\Phi(\cdot, \varrho) V(\varrho, \mathbf{u})} d x=\int_{\Omega} \bar{\Phi} \bar{V} d x \text {, where } \bar{V}=\bar{p}-(2+\nu) \operatorname{div} \mathbf{u} .
$$

Proof. We start with the observation that for every $\delta>0$ there exists a function

$$
\Phi_{\delta}(x, \lambda)=\sum_{k=0}^{n} h_{k}(x) \varphi_{k}(\lambda), \quad h_{k} \in C_{0}^{\infty}(\Omega), \quad \varphi_{k} \in C^{\infty}\left(\mathbb{R}^{+}\right),
$$

such that $\varphi_{k}^{\prime}(\lambda)=0$ for $\lambda>2 N$, and $\left|\Phi_{\delta}-\Phi\right| \leq \delta$. In order to construct $\Phi_{\delta}$ note that there are functions $\psi_{k} \in C^{\infty}(0,2 N), h_{k} \in C_{0}^{\infty}(\Omega), k=1, . ., n$ and $h_{0} \in C_{\Omega}^{\infty}$ such that

$$
\left|\Phi(x, \lambda)-\Phi_{\infty}(x)-\sum_{k=1}^{n} h_{k}(x) \psi_{k}(x)\right| \leq \frac{\delta}{2}, \quad\left|\Phi_{\infty}(x)-h_{0}(x)\right| \leq \frac{\delta}{2}
$$

for all $(x, \lambda) \in \Omega \times[0,2 N]$. Set $\varphi_{0}=1$,

$$
\varphi_{k}=\eta(\lambda) \psi_{k}(\lambda) \text { for } \lambda \leq 2 N, \text { and } \varphi_{k}=0 \text { otherwise }, 1<k \leq n,
$$


where $\eta: \mathbb{R} \mapsto[0,1]$ is a smooth function satisfying the conditions

$$
\eta(\lambda)=1 \quad \text { for } \lambda \leq N, \quad \eta(\lambda)=0 \text { for } \lambda \geq 2 N .
$$

It is clear that the function $\Phi_{\delta}$ defined by 85 , (86) is the desired approximation. Hence it suffices to prove the proposition for $\Phi(x, \lambda)=h(x) \varphi(\lambda)$ with $h \in C_{0}^{\infty}(\Omega)$ and $\varphi \in C^{\infty}(\mathbb{R})$ such that $\varphi^{\prime}(\lambda)=0$ for large $\lambda$.

Denote by $\mathbf{1}_{\Omega}$ the extension operator such that $\mathbf{1}_{\Omega} u=u$ in $\Omega$ and $\mathbf{1}_{\Omega} u=0$ outside $\Omega$. The adjoint operator $\mathbf{1}_{\Omega}^{*}$ assigns to every function its restriction to $\Omega$. Introduce the linear vectorial operator $A$ and the matrix operator $R$ with the components

$$
A_{i}=\mathbf{1}_{\Omega}^{*} \Delta^{-1} \partial_{x_{i}} \mathbf{1}_{\Omega}, \quad R_{i j}=\mathbf{1}_{\Omega}^{*} \partial_{x_{i}} \Delta^{-1} \partial_{x_{j}} \mathbf{1}_{\Omega}, \quad 1 \leq i, j \leq 3 .
$$

Recall that the operators $R_{i j}: L^{r}(\Omega) \mapsto L^{r}(\Omega), A_{i}: L^{r}(\Omega) \mapsto H^{1, r}(\Omega)$ are bounded for every $r>1$. Multiplying regularized moment balance equation (14a) by $h$ we arrive at

$$
\begin{gathered}
h\left(\alpha \varrho_{\varepsilon}+\sqrt{\varepsilon} \varrho_{\varepsilon}^{5}\right) \mathbf{u}_{\varepsilon}+\operatorname{div}\left\{h \mathbf{u}_{\varepsilon} \otimes\left(\varrho_{\varepsilon} \mathbf{u}_{\varepsilon}-\varepsilon \nabla \varrho_{\varepsilon}\right)-\Sigma\left(h \mathbf{u}_{\varepsilon}\right)+h p_{\varepsilon} \mathbf{I}+\mathbf{u}_{\varepsilon} \otimes \nabla h+\right. \\
\left.\nabla h \otimes \mathbf{u}_{\varepsilon}+\nu \nabla h \cdot \mathbf{u}_{\varepsilon} \mathbf{I}\right\}-\left\{\mathbf{u}_{\varepsilon} \otimes\left(\varrho_{\varepsilon} \mathbf{u}_{\varepsilon}-\varepsilon \nabla \varrho_{\varepsilon}\right)-\Sigma\left(\mathbf{u}_{\varepsilon}\right)+p_{\varepsilon} \mathbf{I}\right\} \nabla h=h \varrho_{\varepsilon} \mathbf{F}
\end{gathered}
$$

Next apply to the both sides of this identity the operator $A$ to obtain

$$
\begin{array}{r}
A \cdot\left\{h\left(\alpha \varrho_{\varepsilon}+\sqrt{\varepsilon} \varrho_{\varepsilon}^{5}\right) \mathbf{u}_{\varepsilon}-h \varrho_{\varepsilon} \mathbf{F}\right\}+R:\left\{h \mathbf{u}_{\varepsilon} \otimes\left(\varrho_{\varepsilon} \mathbf{u}_{\varepsilon}-\varepsilon \nabla \varrho_{\varepsilon}\right)-\Sigma\left(h \mathbf{u}_{\varepsilon}\right)+h p_{\varepsilon} \mathbf{I}+\right. \\
\left.2 \mathbf{u}_{\varepsilon} \otimes \nabla h+\nu \nabla h \cdot \mathbf{u}_{\varepsilon} \mathbf{I}\right\}=A \cdot\left\{\left(\mathbf{u}_{\varepsilon} \otimes\left(\varrho_{\varepsilon} \mathbf{u}_{\varepsilon}-\varepsilon \nabla \varrho_{\varepsilon}\right)-\Sigma\left(\mathbf{u}_{\varepsilon}\right)+p_{\varepsilon} \mathbf{I}\right) \nabla h\right\}
\end{array}
$$

Since $h$ is compactly supported in $\Omega$, we have

$$
R:\left\{-\Sigma\left(h \mathbf{u}_{\varepsilon}\right)+h p_{\varepsilon} \mathbf{I}\right\}=h V\left(\varrho_{\varepsilon}, \mathbf{u}_{\varepsilon}\right)-(2+\nu) \nabla h \cdot \mathbf{u}_{\varepsilon}, \quad R:\left(\left(\nabla h \cdot \mathbf{u}_{\varepsilon}\right) \mathbf{I}\right)=\nabla h \cdot \mathbf{u}_{\varepsilon} .
$$

Multiplying the both sides of (87) by $\varphi_{\varepsilon}=\varphi\left(\varrho_{\varepsilon}\right)$, integrating the result over $\Omega$, and using relations (88) we obtain

$$
\begin{gathered}
\int_{\Omega} h \varphi_{\varepsilon} V\left(\varrho_{\varepsilon}, \mathbf{u}_{\varepsilon}\right) d x+\int_{\Omega} \varphi_{\varepsilon}\left(\mathcal{P}_{\varepsilon}+\mathcal{Q}_{\varepsilon}+R:\left(h \varrho_{\varepsilon} \mathbf{u}_{\varepsilon} \otimes \mathbf{u}_{\varepsilon}\right)\right) d x=0, \\
\mathcal{P}_{\varepsilon}=-2 \nabla h \cdot \mathbf{u}_{\varepsilon}+2 R:\left(\mathbf{u}_{\varepsilon} \otimes \nabla h\right)- \\
A \cdot\left\{\left(\varrho_{\varepsilon} \mathbf{u}_{\varepsilon} \otimes \mathbf{u}_{\varepsilon}-\Sigma\left(\mathbf{u}_{\varepsilon}\right)+p_{\varepsilon} \mathbf{I}\right) \nabla h+\varrho_{\varepsilon} h\left(\mathbf{F}-\alpha \mathbf{u}_{\varepsilon}\right)\right\} \\
\mathcal{Q}_{\varepsilon}=A \cdot\left\{h \sqrt{\varepsilon} \varrho_{\varepsilon}^{5} \mathbf{u}_{\varepsilon}+\left(\varepsilon \mathbf{u}_{\varepsilon} \otimes \nabla \varrho_{\varepsilon}\right) \nabla h\right\}-R:\left(\varepsilon h \mathbf{u}_{\varepsilon} \otimes \nabla \varrho_{\varepsilon}\right) .
\end{gathered}
$$

On the other hand, multiplying the both sides of regularized mass balance equation 14b by $h \varphi^{\prime}\left(\varrho_{\varepsilon}\right)$ we get

$$
\begin{gathered}
h \varphi_{\varepsilon}^{\prime}\left(\varrho_{\varepsilon}\right)\left(\alpha \varrho_{\varepsilon}+\sqrt{\varepsilon} \varrho_{\varepsilon}^{5}\right)+\operatorname{div}\left(h \varphi_{\varepsilon} \mathbf{u}_{\varepsilon}\right)+h\left(\varphi_{\varepsilon}^{\prime}\left(\varrho_{\varepsilon}\right) \varrho_{\varepsilon}-\varphi_{\varepsilon}\right) \operatorname{div} \mathbf{u}_{\varepsilon}-\varphi_{\varepsilon} \nabla h \cdot \mathbf{u}_{\varepsilon}= \\
\varepsilon\left[\Delta\left(h \varphi_{\varepsilon}\right)-2 \nabla h \cdot \nabla \varphi_{\varepsilon}-\varphi_{\varepsilon} \Delta h-h \varphi^{\prime \prime}\left(\varrho_{\varepsilon}\right)\left|\nabla \varrho_{\varepsilon}\right|^{2}\right]+h \varphi^{\prime}(\varepsilon) f .
\end{gathered}
$$


Introduce the vector field

$$
\mathbf{v}_{\varepsilon}=\varrho_{\varepsilon}^{(n)} \mathbf{u}_{\varepsilon} \text { where } \varrho_{\varepsilon}^{(n)}=\min \{n, \varrho\}, n \geq 1 .
$$

Applying the operator $\mathbf{v}_{\varepsilon} \cdot A:=v_{\varepsilon, i} A_{i}$ to the both sides of (90), integrating the result over $\Omega$, and using the identities

$\int_{\Omega} \mathbf{v}_{\varepsilon} \cdot A \Delta\left(h \varphi_{\varepsilon}\right) d x=\int_{\Omega} \nabla\left(h \varphi_{\varepsilon}\right) R \mathbf{v}_{\varepsilon} d x, \quad \int_{\Omega} \mathbf{v}_{\varepsilon} \cdot A\left(h \varphi^{\prime \prime}\left(\varrho_{\varepsilon}\right)\left|\nabla \varrho_{\varepsilon}\right|^{2}\right) d x=-\int_{\Omega} h \varphi^{\prime \prime}\left(\varrho_{\varepsilon}\right)\left|\nabla \varrho_{\varepsilon}\right|^{2} A \cdot \mathbf{v}_{\varepsilon} d x$

we arrive at the equality

$$
\begin{gathered}
\int_{\Omega}\left\{\mathbf{v}_{\varepsilon}\left(\mathfrak{P}_{\varepsilon}+\mathfrak{Q}_{\varepsilon}\right)-h\left(A \cdot \mathbf{v}_{\varepsilon}\right) I_{\varepsilon}\right\} d x+\int_{\Omega} \mathbf{v}_{\varepsilon} R\left(h \varphi_{\varepsilon} \mathbf{u}_{\varepsilon}\right) d x=0, \\
\mathfrak{P}_{\varepsilon}=A \cdot\left\{\alpha h \varphi^{\prime}\left(\varrho_{\varepsilon}\right) \varrho_{\varepsilon}+h\left(\varphi^{\prime}\left(\varrho_{\varepsilon}\right) \varrho_{\varepsilon}-\varphi_{\varepsilon}\right) \operatorname{div} \mathbf{u}_{\varepsilon}-\varphi_{\varepsilon} \nabla h \cdot \mathbf{u}_{\varepsilon}-h \varphi^{\prime}\left(\varrho_{\varepsilon}\right) f\right\}, \\
\mathfrak{Q}_{\varepsilon}=A \cdot\left\{2 \varepsilon \nabla h \nabla \varphi_{\varepsilon}+\varepsilon \varphi_{\varepsilon} \Delta h+h \sqrt{\varepsilon} \varrho_{\varepsilon}^{5}\right\}-\varepsilon R \nabla\left(h \varphi_{\varepsilon}\right), \\
I_{\varepsilon}=\varepsilon \varphi^{\prime \prime}\left(\varrho_{\varepsilon}\right)\left|\nabla \varrho_{\varepsilon}\right|^{2} .
\end{gathered}
$$

Combining (89), (91) and noting that

$$
\begin{gathered}
\int_{\Omega}\left(\mathbf{v}_{\varepsilon} R\left(h \varphi_{\varepsilon} \mathbf{u}_{\varepsilon}\right)-\varphi_{\varepsilon} R:\left(h \varrho_{\varepsilon} \mathbf{u}_{\varepsilon} \otimes \mathbf{u}_{\varepsilon}\right)\right) d x=\int_{\Omega}\left(\mathbf{v}_{\varepsilon i} R_{i j}\left(h \varphi_{\varepsilon} \mathbf{u}_{\varepsilon j}\right)-\varphi_{\varepsilon} R_{i j}\left(h \mathbf{v}_{\varepsilon i} \mathbf{u}_{\varepsilon j}\right)\right) d x+ \\
\int_{\Omega} \varphi_{\varepsilon} R:\left[\left(\varrho_{\varepsilon}^{(n)}-\varrho_{\varepsilon}\right) h \mathbf{u}_{\varepsilon} \otimes \mathbf{u}_{\varepsilon}\right] d x=\int_{\Omega} h u_{\varepsilon j}\left(\varphi_{\varepsilon} R_{i j} \mathbf{v}_{\varepsilon i}-\mathbf{v}_{\varepsilon i} R_{i j} \varphi_{\varepsilon}\right) d x+ \\
\int_{\Omega} \varphi_{\varepsilon} R:\left[\left(\varrho_{\varepsilon}^{(n)}-\varrho_{\varepsilon}\right) h \mathbf{u}_{\varepsilon} \otimes \mathbf{u}_{\varepsilon}\right] d x .
\end{gathered}
$$

we obtain the equality

$$
\int_{\Omega} h \varphi_{\varepsilon} V\left(\varrho_{\varepsilon}, \mathbf{u}_{\varepsilon}\right) d x=\int_{\Omega}\left(\mathbf{v}_{\varepsilon}\left(\mathfrak{P}_{\varepsilon}+\mathfrak{Q}_{\varepsilon}\right)-\varphi_{\varepsilon}\left(\mathcal{P}_{\varepsilon}+\mathcal{Q}_{\varepsilon}\right)+\mathfrak{R}_{\varepsilon}-h\left(A \cdot \mathbf{v}_{\varepsilon}\right) I_{\varepsilon}\right) d x+\varpi_{\varepsilon},
$$

in which components of the vector $\mathfrak{R}_{\varepsilon}$ and the scalar $\varpi_{\varepsilon}$ defined by

$$
\Re_{\varepsilon, i}=h \mathbf{u}_{\varepsilon, j}\left(\varphi_{\varepsilon} R_{i j} \mathbf{v}_{\varepsilon i}-\mathbf{v}_{\varepsilon i} R_{i j} \varphi_{\varepsilon}\right), \quad \varpi_{\varepsilon}=\int_{\Omega} \varphi_{\varepsilon} R:\left[\left(\varrho_{\varepsilon}^{(n)}-\varrho_{\varepsilon}\right) h \mathbf{u}_{\varepsilon} \otimes \mathbf{u}_{\varepsilon}\right] d x
$$

$\mathrm{RR} \mathrm{n}^{\circ} 5481$ 
Recall that $\varrho_{\varepsilon} \mathbf{u}_{\varepsilon} \otimes \mathbf{u}_{\varepsilon} \nabla h \rightarrow \varrho \mathbf{u} \otimes \mathbf{u} \nabla h$ and $p_{\varepsilon} \nabla h \rightarrow \bar{p} \nabla h$ weakly in $L^{1+\kappa}(\Omega)$ as $\varepsilon \rightarrow 0$. Hence,

$$
\begin{aligned}
& \mathcal{P}_{\varepsilon} \rightarrow \overline{\mathcal{P}} \equiv- 2 \nabla h \mathbf{u}+2 R:(\mathbf{u} \otimes \nabla h)-A \cdot\{(\varrho \mathbf{u} \otimes \mathbf{u}-\Sigma(\mathbf{u})+\bar{p} \mathbf{I}) \nabla h+ \\
&h \varrho(\mathbf{F}-\alpha \mathbf{u})\} \quad \text { in } L^{r}(\Omega) \text { for some } r>1, \\
& \mathfrak{P}_{\varepsilon} \rightarrow \overline{\mathfrak{P}} \equiv A \cdot\left\{\alpha h \overline{\varphi \varrho}+h \overline{\left(\varphi^{\prime} \varrho-\varphi\right) \operatorname{div} \mathbf{u}}-\bar{\varphi} \nabla h \cdot \mathbf{u}-\overline{\varphi^{\prime}} h f\right\} \text { in } L^{2}(\Omega) .
\end{aligned}
$$

Estimates (73) yield

$$
\mathcal{Q}_{\varepsilon} \rightarrow 0 \text { in } L^{1}(\Omega) \text { and } \mathfrak{Q}_{\varepsilon} \rightarrow 0 \text { in } L^{2}(\Omega) \text { as } \varepsilon \rightarrow 0 .
$$

Next, the functions $I_{\varepsilon}$ are uniformly bounded in $L^{1}(\Omega)$ and converge weakly in the space of Radon measures $C_{0}^{*}(\Omega)$ to the Radon measure $M_{\varphi}$ defined by

$$
\left\langle M_{\varphi}, h\right\rangle=\int_{\Omega \times[0, \infty)} h(x) \varphi^{\prime \prime}(\lambda) d m(x, \lambda),
$$

where the measure $m$ is given by $(80)$. Since the sequence $\mathbf{v}_{\varepsilon}$ converges weakly in $L^{6}(\Omega)$, the continuous functions $A \cdot \mathbf{v}_{\varepsilon}$ converge uniformly in $\Omega$ to $A \cdot \overline{\mathbf{v}}$ which leads to

$$
\lim _{\varepsilon \rightarrow 0} \int_{\Omega} h\left(A \cdot \mathbf{v}_{\varepsilon}\right) I_{\varepsilon} d x=\left\langle M_{\varphi}, h A \cdot \overline{\mathbf{v}}\right\rangle .
$$

Since the sequences $\varphi_{\varepsilon}$ and $\mathbf{v}_{\varepsilon}$ are bounded in $L^{\infty}(\Omega)$ and $L^{6}(\Omega)$, respectively, it follows from the compensated compactness Lemma from [14] that

$$
\varphi_{\varepsilon} R_{i j} \mathbf{v}_{\varepsilon i}-\mathbf{v}_{\varepsilon i} R_{i j} \varphi_{\varepsilon} \rightarrow \bar{\varphi} R_{i j} \overline{\mathbf{v}}_{i}-\overline{\mathbf{v}}_{i} R_{i j} \bar{\varphi} \text { weakly in } L^{2}(\Omega) .
$$

Therefore, $\mathfrak{R}_{\varepsilon}$ converges weakly in $L^{3 / 2}(\Omega)$ to $\bar{\Re}=\left\{\mathbf{u}_{j}\left(\bar{\varphi} R_{i j} \overline{\mathbf{v}}_{i}-\overline{\mathbf{v}}_{i} R_{i j} \bar{\varphi}\right)\right\}$. Passing to the limit in (92) and using (94) -97) we obtain

$$
\int_{\Omega} h \overline{\varphi V} d x=\int_{\Omega}(\overline{\mathbf{v}} \overline{\mathfrak{P}}-\bar{\varphi} \overline{\mathcal{P}}+\overline{\mathfrak{R}}) d x-\int_{\Omega} h A \cdot \overline{\mathbf{v}} d M_{\varphi}(x)+\bar{\varpi}
$$

with $|\varpi| \leq \limsup \left|\varpi_{\varepsilon}\right|$. On the other hand, passage to the limit in equalities (87) and (90) gives

$$
\begin{array}{r}
h \alpha \varrho+\operatorname{div}\{h \varrho \mathbf{u} \otimes \mathbf{u}-\Sigma(h \mathbf{u})+h \bar{p} I+ \\
\mathbf{u} \otimes \nabla h+\nabla h \otimes \mathbf{u}+\nabla h \cdot \mathbf{u}\}-(\varrho \mathbf{u} \otimes \mathbf{u}-\Sigma(\mathbf{u})+\bar{p} I) \nabla h=h \varrho \mathbf{F}, \\
\alpha h \overline{\varphi^{\prime} \varrho}+\operatorname{div}(h \bar{\varphi} \mathbf{u})+h \overline{\left(\varphi^{\prime} \varrho-\varphi\right) \operatorname{div} \mathbf{u}}-\bar{\varphi} \nabla h \cdot \mathbf{u}+h M_{\varphi}=\overline{\varphi^{\prime}} f
\end{array}
$$


Applying to the both sides of (99), (100) the operators $\bar{\varphi} A$ and $\overline{\mathbf{v}} \cdot A$, respectively, and arguing as before we obtain

$$
\int_{\Omega} h \bar{\varphi} \bar{V} d x=\int_{\Omega}(\overline{\mathbf{v}} \overline{\mathfrak{P}}-\bar{\varphi} \overline{\mathcal{P}}+\overline{\mathfrak{R}}) d x+\int_{\Omega} h A \cdot \overline{\mathbf{v}} d M_{\varphi}(x)+\varpi,
$$

where

$$
\varpi=\int_{\Omega} \bar{\varphi} R:[h(\overline{\mathbf{v}}-\varrho \mathbf{u}) \otimes \mathbf{u}] d x .
$$

Combining (98) and (101) we finally obtain

$$
\int_{\Omega} h \bar{\varphi} \bar{V} d x-\int_{\Omega} h \overline{\varphi V} d x=\varpi-\bar{\varpi} .
$$

By Lemma 6.4 the sequence $h \varrho_{\varepsilon} \mathbf{u}_{\varepsilon} \otimes \mathbf{u}_{\varepsilon}$ converges weakly in $L^{1+\kappa}(\Omega)$ to $h \varrho \mathbf{u} \otimes \mathbf{u}$; obviously $\varrho_{\varepsilon}^{(n)} \mathbf{u}_{\varepsilon}=\mathbf{v}_{\varepsilon}$ converges weakly in $L^{6}(\Omega)$ to $\overline{\mathbf{v}}$. From this we conclude that

$$
\begin{gathered}
|\varpi| \leq\|R:[h(\overline{\mathbf{v}}-\varrho \mathbf{u}) \otimes \mathbf{u}]\|_{L^{1}(\Omega)} \leq c\|h(\overline{\mathbf{v}}-\varrho \mathbf{u}) \otimes \mathbf{u}\|_{L^{1+\kappa / 2}(\Omega)} \leq \\
c \underset{\varepsilon \rightarrow 0}{\limsup }\left\|h\left(\mathbf{v}_{\varepsilon}-\varrho_{\varepsilon} \mathbf{u}_{\varepsilon}\right) \otimes \mathbf{u}_{\varepsilon}\right\|_{L^{1+\kappa / 2}(\operatorname{spt} h)} .
\end{gathered}
$$

The similar arguments give the same bound for $\limsup _{\varepsilon \rightarrow 0}\left|\varpi_{\varepsilon}\right|$. Since the sequence $\varrho_{\varepsilon} \mathbf{u}_{\varepsilon} \otimes \mathbf{u}_{\varepsilon}$ is bounded in $L_{l o c}^{1+\kappa}(\Omega)$ and the sequence $\varrho_{\varepsilon}$ is bounded in $L^{1}(\Omega)$, we have

$$
\begin{gathered}
\left\|h\left(\mathbf{v}_{\varepsilon}-\varrho_{\varepsilon} \mathbf{u}_{\varepsilon}\right) \otimes \mathbf{u}_{\varepsilon}\right\|_{L^{1+\kappa / 2}(\mathrm{spt} h)} \leq c(h)\left\|\left(\varrho_{\varepsilon}^{(n)}-\varrho_{\varepsilon}\right) \mathbf{u}_{\varepsilon} \otimes \mathbf{u}_{\varepsilon}\right\|_{L^{1+\kappa / 2}(\mathrm{spt} h)} \leq \\
c\left(\int_{\left\{\varrho_{\varepsilon}>n\right\} \cap \operatorname{spt} h}\left(\varrho_{\varepsilon}\left|\mathbf{u}_{\varepsilon}\right|^{2}\right)^{1+\kappa / 2} d x\right)^{2 /(2+\kappa)} \leq c\left\|\varrho_{\varepsilon}\left|\mathbf{u}_{\varepsilon}\right|^{2}\right\|_{L^{1+\kappa}(\mathrm{spt} h)} \operatorname{mes}\left\{\varrho_{\varepsilon}<n\right\}^{\iota} \leq c n^{-\iota},
\end{gathered}
$$

where $\iota=\kappa(1+\kappa)^{-1}(2+\kappa)^{-1}>0$. Hence $|\varpi|+|\bar{\varpi}| \rightarrow 0$ as $n \rightarrow \infty$. It remains to note that the left side of (102) does not depend on $n$, and the lemma follows.

The oscillation defect measure The notion of oscillation defect measure was introduced in 12 in order to justify the existence theory for isentropic flows with "small" values of the adiabatic constant $\gamma$. Following [12, 15] the oscillation defect measure associated with the sequence $\varrho_{\varepsilon}$ is defined as follows

$$
\operatorname{osc}_{p}\left[\varrho_{\varepsilon} \rightarrow \varrho\right](\Omega):=\sup _{k \geq 1}\left(\limsup _{\varepsilon \rightarrow 0} \int_{\Omega}\left|T_{k}\left(\varrho_{\varepsilon}\right)-T_{k}(\varrho)\right|^{p} d x\right),
$$

where $T_{k}(z)=k T(z / k), T(z)$ is a smooth concave function, which is equal to $z$ for $z \leq 1$ and is a const for $z \geq 3$. The smoothness properties of $T_{k}$ are not important and we can take it 
in the simplest form $T_{k}(z)=\min \{z, k\}$. In particular, for the sequence of $\varrho_{\varepsilon}$ satisfying the hypotheses of Lemma 7.1 we have

$$
\mathbf{o s c}_{p}\left[\varrho_{\varepsilon} \rightarrow \varrho\right](\Omega)=\sup _{k \geq 1} \lim _{\varepsilon \rightarrow 0} \int_{\Omega}\left|\min \left\{\varrho_{\varepsilon}, k\right\}-\min \{\varrho, k\}\right|^{p} d x \geq \sup _{k \geq 1} \int_{\Omega}\left|\mathcal{T}_{k}\right|^{p} d x,
$$

where $\mathcal{T}_{k}=\overline{\min \{\varrho, k\}}-\min \{\varrho, k\}$. The important consequence of Lemma 7.3 is the following version of the result of E. Feireisl et al [12, 14] on the integrability of the oscillations defect measure. In order to formulate the result we introduce the function $\mathcal{T}_{\vartheta}(x)$ defined by the equality $\mathcal{T}_{\vartheta}(x)=\overline{\min \{\varrho, \vartheta\}}(x)-\min \{\varrho(x), \vartheta(x)\}$ for any $\vartheta \in C(\Omega)$.

Lemma 7.4 Under the assumptions of Theorem [1.1 and Lemma 7.1, there is a constant c independent of $\vartheta$ such that the inequalities

$$
\left\|\mathcal{T}_{\vartheta}\right\|_{L^{1+\gamma}(\Omega)}^{1+\gamma} \leq \lim _{\varepsilon \rightarrow 0} \int_{\Omega}\left|\min \left\{\varrho_{\varepsilon}(x), \vartheta(x)\right\}-\min \{\varrho(x), \vartheta(x)\}\right|^{1+\gamma} d x \leq c
$$

hold for all $\vartheta \in C(\Omega)$. Recall that the limit in the right side exists by the choice of the sequence $\varrho_{\varepsilon}$.

Proof. The proof imitates the proof of Lemma 4.3 from [14]. It is easily seen that

$$
\left\|\mathcal{T}_{\vartheta}\right\|_{L^{1+\gamma}(\Omega)}^{1+\gamma} \leq \limsup _{\varepsilon \rightarrow 0} \int_{\Omega}\left|\min \left\{\varrho_{\varepsilon}(x), \vartheta(x)\right\}-\min \{\varrho(x), \vartheta(x)\}\right|^{1+\gamma} d x .
$$

Hence, it suffices to show that the right side of this inequality has a bound independent of $\vartheta$. Noting that

$$
\left|\min \left\{s^{\prime}, \vartheta\right\}-\min \left\{s^{\prime \prime}, \vartheta\right\}\right|^{1+\gamma} \leq\left(\min \left\{s^{\prime}, \vartheta\right\}-\min \left\{s^{\prime \prime}, \vartheta\right\}\right)\left(s^{\prime \gamma}-s^{\prime \prime \gamma}\right) \text { for all } s^{\prime}, s^{\prime \prime} \in \mathbb{R}^{+},
$$

and $\overline{\varrho^{\gamma}} \geq \varrho^{\gamma}, \overline{\min \{\varrho, \vartheta\}} \leq \min \{\varrho, \vartheta\}$ we get for any compactly supported, non-negative function $h \in C(\Omega)$,

$$
\begin{aligned}
& \lim _{\varepsilon \rightarrow 0} \int_{\Omega} h\left|\min \left\{\varrho_{\varepsilon}, \vartheta\right\}-\min \{\varrho, \vartheta\}\right|^{1+\gamma} d x \leq \lim _{\varepsilon \rightarrow 0} \int_{\Omega} h\left(\min \left\{\varrho_{\varepsilon}, \vartheta\right\}-\min \{\varrho, \vartheta\}\right)\left(\varrho_{\varepsilon}^{\gamma}-\varrho^{\gamma}\right) d x \leq \\
& \lim _{\varepsilon \rightarrow 0} \int_{\Omega} h\left(\min \left\{\varrho_{\varepsilon}, \vartheta\right\}-\min \{\varrho, \vartheta\}\right)\left(\varrho_{\varepsilon}^{\gamma}-\varrho^{\gamma}\right) d x+\int_{\Omega}\left(\overline{\varrho^{\gamma}}-\varrho^{\gamma}\right)(\min \{\varrho, \vartheta\}-\overline{\min \{\varrho, \vartheta\}}) d x= \\
& \lim _{\varepsilon \rightarrow 0} \int_{\Omega} h\left(\varrho_{\varepsilon}^{\gamma} \min \left\{\varrho_{\varepsilon}, \vartheta\right\}-\overline{\varrho^{\gamma}} \overline{\min \{\varrho, \vartheta\}}\right) d x=\lim _{\varepsilon \rightarrow 0} \int_{\Omega} h\left(p_{\varepsilon} \min \left\{\varrho_{\varepsilon}, \vartheta\right\}-\bar{p} \overline{\min \{\varrho, \vartheta\}}\right) d x .
\end{aligned}
$$


By Lemma 7.3 with $\Phi(\varrho, x)=h(x) \min \{\varrho, \vartheta(x)\}$, the right side of (105), divided by $(2+\nu)$, is equal to

$$
\begin{gathered}
\lim _{\varepsilon \rightarrow 0} \int_{\Omega} h\left(\min \left\{\varrho_{\varepsilon}, \vartheta\right\} \operatorname{div} \mathbf{u}_{\varepsilon}-\overline{\min \{\varrho, \vartheta\}} \operatorname{div} \mathbf{u}\right) d x= \\
\lim _{\varepsilon \rightarrow 0} \int_{\Omega} h\left(\min \left\{\varrho_{\varepsilon}, \vartheta\right\}-\min \{\varrho, \vartheta\}\right) \operatorname{div} \mathbf{u}_{\varepsilon} d x-\lim _{\varepsilon \rightarrow 0} \int_{\Omega} h\left(\min \left\{\varrho_{\varepsilon}, \vartheta\right\}-\min \{\varrho, \vartheta\}\right) \operatorname{div} \mathbf{u} d x \\
\leq \delta \limsup _{\varepsilon \rightarrow 0} \int_{\Omega} h\left|\min \left\{\varrho_{\varepsilon}, \vartheta\right\}-\min \{\varrho, \vartheta\}\right|^{1+\gamma} d x+\delta^{-\gamma} \limsup _{\varepsilon \rightarrow 0} \int_{\Omega} h\left(\left|\operatorname{div} \mathbf{u}_{\varepsilon}\right|+|\operatorname{div} \mathbf{u}|\right)^{(1+\gamma) / \gamma} \leq \\
\delta \limsup _{\varepsilon \rightarrow 0} \int_{\Omega} h\left|\min \left\{\varrho_{\varepsilon}, \vartheta\right\}-\min \{\varrho, \vartheta\}\right|^{1+\gamma} d x+c \delta^{-\gamma}\|h\|_{C(\Omega)} .
\end{gathered}
$$

Combining (106) and (105), letting $h \rightarrow 1$, and choosing $\delta$ sufficiently small gives (103)

We reformulate this result in terms of the distribution function $\Gamma$. Recall that the functions $\min \left\{\varrho_{\varepsilon}, \lambda\right\}$ are uniformly bounded and $\min \left\{\varrho_{\varepsilon}, \lambda\right\} \operatorname{div} \mathbf{u}_{\varepsilon}$ converges weakly in $L^{2}(\Omega)$ for each non-negative $\lambda$. Introduce the functions

$$
\begin{gathered}
\mathcal{V}_{\lambda}=\overline{(\min \{\varrho, \lambda\} \operatorname{div} \mathbf{u})}-\overline{\min \{\varrho, \lambda\}} \operatorname{div} \mathbf{u} \in L^{2}(\Omega), \\
\mathfrak{H}=\int_{[0, \infty)} \Gamma(x, s)(1-\Gamma(x, s)) d s, \quad x \in \Omega .
\end{gathered}
$$

Lemma 7.5 There is a constant $c$ independent of $\lambda$ such that

$$
\|\mathfrak{H}\|_{L^{1+\gamma}(\Omega)}+\sup _{\lambda}\left\|\mathcal{V}_{\lambda}\right\|_{L^{1}(\Omega)} \leq c .
$$

Proof. We begin with the observation that by Lemma [7.1]

$$
\mathcal{T}_{\vartheta}(x)=\int_{[0, \infty)} \min \{\lambda, \vartheta(x)\} d_{\lambda} \Gamma(x, \lambda)-\min \left\{\int_{[0, \infty)} \lambda d_{\lambda} \Gamma(x, \lambda), \vartheta\right\}
$$

for each $\vartheta \in C(\Omega)$. From this and the identity $\varrho(x)=\int_{[0, \infty)}(1-\Gamma(x, \lambda)) d \lambda$ we conclude that

$$
\mathcal{T}_{\vartheta}(x)=\int_{0}^{\vartheta(x)} \Gamma(x, s) d s \text { for } \vartheta(x) \geq \varrho(x) \text { and } \mathcal{T}_{\vartheta}(x)=\int_{\vartheta(x)}^{\infty}(1-\Gamma(x, s)) d s \text { otherwise. }
$$


Next choose a sequence of continuous non-negative functions $\vartheta_{k} \rightarrow \varrho$ as $k \rightarrow \infty$ a.e. in $\Omega$. By Lemma 7.4, the functions $\mathcal{T}_{\vartheta_{k}}$ are uniformly bounded in $L^{1+\gamma}(\Omega)$ and converges a.e. in $\Omega$ to the function

$$
\mathcal{T}_{\varrho}(x)=\int_{0}^{\varrho(x)} \Gamma(x, s) d s=\int_{\varrho(x)}^{\infty}(1-\Gamma(x, s)) d s,
$$

which yields the inclusion $\mathcal{T}_{\varrho} \in L^{1+\gamma}(\Omega)$. It remains to note that estimate (108) for $\mathfrak{H}$ obviously follows from the inequality $\mathfrak{H} \leq 2 \mathcal{T}_{\varrho}$.

In order to estimate $\mathcal{V}_{\lambda}$ note that

$$
\mathcal{V}_{\lambda}=\mathrm{w}-\lim _{\varepsilon \rightarrow 0}\left(\left(\min \left\{\varrho_{\varepsilon}, \lambda\right\}-\min \{\varrho, \lambda\}\right) \operatorname{div} \mathbf{u}_{\varepsilon}\right)-\left(\mathrm{w}-\lim _{\varepsilon \rightarrow 0} \min \left\{\varrho_{\varepsilon}, \lambda\right\}-\min \{\varrho, \lambda\}\right) \operatorname{div} \mathbf{u},
$$

where $w-\lim$ is the weak limit in $L^{1}(\Omega)$. From this and the boundedness of norms $\left\|\operatorname{div} \mathbf{u}_{\varepsilon}\right\|_{L^{2}(\Omega)}$ we obtain

$$
\begin{gathered}
\left\|\mathcal{V}_{\lambda}\right\|_{L^{1}(\Omega)} \leq \limsup _{\varepsilon \rightarrow 0}\left(\left\|\operatorname{div} \mathbf{u}_{\varepsilon}\right\|_{L^{2}(\Omega)}+\right. \\
\left.\|\operatorname{div} \mathbf{u}\|_{L^{2}(\Omega)}\right)\left\|\min \left\{\varrho_{\varepsilon}(x), \lambda\right\}-\min \{\varrho(x), \lambda\}\right\|_{L^{2}(\Omega)},
\end{gathered}
$$

which along with (103) yields (108) and the lemma follows.

\section{Proof of Theorem 6.5}

It follows from Remark 7.2 that Theorem 6.5 will be proved if we show that $\Gamma(x, \lambda)(1-$ $\Gamma(x, \lambda))=0$ almost everywhere in $\Omega \times \mathbb{R}$. We split the proof of this equality into three steps. First, the kinetic equation for the distribution function is derived.

Kinetic formulation of the mass balance equation In this paragraph we show that the distribution function $\Gamma(x, \lambda)$ of the Young measure, associated with a sequence of solutions to problem (14), satisfies some integro-differential transport equation named a kinetic equation. This result is given by the following lemma.

Lemma 8.1 Suppose that all assumptions of Theorem [6.5 are satisfied and $\Gamma$ is a distribution function of the Young measure associated with a sequence $\varrho_{\varepsilon}$ of solutions to problem (14). Then for any $\zeta \in C_{0}^{\infty}(\Omega \times \mathbb{R})$,

$$
\begin{gathered}
\int_{\Omega \times \mathbb{R}_{\lambda}} \zeta\{\lambda \alpha-f(x)\} d_{\lambda} \Gamma(x, \lambda) d x+\int_{\Omega \times \mathbb{R}_{\lambda}} \Gamma(x, \lambda) \nabla_{x, \lambda} \zeta \cdot \mathbf{w} d \lambda d x+ \\
\int_{\Omega \times \mathbb{R}_{\lambda}} \partial_{\lambda} \zeta d m(x, \lambda)+\int_{\Omega \times \mathbb{R}_{\lambda}} \partial_{\lambda} \zeta \lambda M(x, \lambda) d x=0 .
\end{gathered}
$$


Here $m$ is the Radon measure on $\Omega \times \mathbb{R}_{\lambda}$ given by (81), the solenoidal vector field $\mathbf{w}=$ $(\mathbf{u}(x),-\lambda$ div $\mathbf{u})$, and the function $M$ is defined by the equalities

$$
M=-\frac{1}{2+\nu} \int_{(-\infty, \lambda)}\left(s^{\gamma}-\bar{p}\right) d_{s} \Gamma(x, s)=\frac{1}{2+\nu} \int_{[\lambda, \infty)}\left(s^{\gamma}-\bar{p}\right) d_{s} \Gamma(x, s),
$$

in which $\bar{p}(x)=\int_{\mathbb{R}} p(\lambda) d_{\lambda} \Gamma(x, \lambda)$. For almost every $x$ the non-negative function $M(x, \cdot)$ has a bounded variation $\operatorname{Var}_{\lambda} M(x, \cdot)$, which belongs to $L^{1}(\Omega)$. Note that integral identity (111) is equivalent to kinetic equation (17), which is understood in the sense of distributions.

Proof. Choose an arbitrary $\varphi \in C^{\infty}(\mathbb{R})$ vanishing near $+\infty$, and note that for an arbitrary $h \in C_{0}^{\infty}(\Omega)$,

$$
\begin{gathered}
\int_{\Omega}\left\{h \varphi_{\varepsilon}^{\prime}\left(\varrho_{\varepsilon}\right)\left(\alpha \varrho_{\varepsilon}+\sqrt{\varepsilon} \varrho_{\varepsilon}^{5}-f\right)-\varphi_{\varepsilon} \nabla h \cdot \mathbf{u}_{\varepsilon}+h\left(\varphi_{\varepsilon}^{\prime}\left(\varrho_{\varepsilon}\right) \varrho_{\varepsilon}-\varphi_{\varepsilon}\right) \operatorname{div} \mathbf{u}_{\varepsilon}\right\} d x+ \\
\int_{\Omega} \varepsilon\left\{-\Delta h \varphi_{\varepsilon}+h \varphi^{\prime \prime}\left(\varrho_{\varepsilon}\right)\left|\nabla \varrho_{\varepsilon}\right|^{2}\right\} d x=0 .
\end{gathered}
$$

Letting $\varepsilon \rightarrow 0$ we obtain

$$
\begin{gathered}
\int_{\Omega}\left(\alpha \overline{\varphi^{\prime} \varrho} h-\bar{\varphi} \nabla h \cdot \mathbf{u}-h f \overline{\varphi^{\prime}}\right) d x+ \\
\int_{\Omega} h \overline{\left(\varphi^{\prime}(\varrho) \varrho-\varphi\right) \operatorname{div} \mathbf{u}} d x+\int_{\Omega \times \mathbb{R}_{\lambda}} h(x) \varphi^{\prime \prime}(\lambda) d m(x, \lambda)=0 .
\end{gathered}
$$

Lemma 7.3 gives the expression for the second integral at the right side

$$
\int_{\Omega} h \overline{\left(\varphi^{\prime}(\varrho) \varrho-\varphi\right) \operatorname{div} \mathbf{u}} d x=\int_{\Omega} h\left[\overline{\left(\varphi^{\prime} \varrho-\varphi\right)} \operatorname{div} \mathbf{u}+\frac{1}{2+\nu}\left(\overline{\left(\varphi^{\prime} \varrho-\varphi\right) p}-\overline{\left(\varphi^{\prime} \varrho-\varphi\right)} \bar{p}\right)\right] d x .
$$

Next choose an arbitrary smooth function $\eta(\lambda)$ vanishing near $+\infty$ and set $\varphi(\lambda)=\int_{\lambda}^{\infty} \eta(s) d s$. Lemma 7.1 yields the representations

$$
\begin{gathered}
\overline{\varphi^{\prime} \varrho}(x)=-\int_{[0, \infty)} \lambda \eta(\lambda) d_{\lambda} \Gamma(x, \lambda), \quad \overline{\varphi^{\prime}}(x)=-\int_{[0, \infty)} \eta(\lambda) d_{\lambda} \Gamma(x, \lambda), \\
\bar{\varphi}(x)=\int_{[0, \infty)}\left\{\int_{\lambda}^{\infty} \eta(s) d s\right\} d_{\lambda} \Gamma(x, \lambda)=\int_{[0, \infty)} \eta(\lambda) \Gamma(x, \lambda) d \lambda,
\end{gathered}
$$

$\mathrm{RR} \quad \mathrm{n}^{\circ} 5481$ 
which are substituted into (113) and (114) and lead to the equality

$$
\begin{gathered}
\int_{\Omega \times \mathbb{R}_{\lambda}} h \eta\{f-\lambda \alpha-\lambda \operatorname{div} \mathbf{u}\} d_{\lambda} \Gamma d x-\int_{\Omega \times \mathbb{R}_{\lambda}} \eta \operatorname{div}(h \mathbf{u}) \Gamma d x d \lambda- \\
\int_{\Omega \times \mathbb{R}_{\lambda}} h \eta^{\prime} d m(x, t)+\frac{1}{2+\nu} \int_{\Omega} h\left(\overline{\left(\varphi^{\prime} \varrho-\varphi\right) p}-\overline{\left(\varphi^{\prime} \varrho-\varphi\right)} \bar{p}\right) d x
\end{gathered}
$$

Recall that $\Gamma$ and $m$ vanish for $\lambda<0$, the function $G(\lambda, x)=\left(\varphi^{\prime}(\lambda) \lambda-\varphi(\lambda)\right) h(x)$ satisfies all conditions of Lemma 7.1 and $h$ is compactly supported in $\Omega$. It follows from this and Lemma 7.1 that

$$
\begin{array}{r}
\int_{\Omega} h\left(\overline{\left(\varphi^{\prime} \varrho-\varphi\right) p}-\overline{\left(\varphi^{\prime} \varrho-\varphi\right)} \bar{p}\right) d x=-\int_{\Omega \times \mathbb{R}_{\lambda}} h \eta \lambda\left(\lambda^{\gamma}-\bar{p}\right) d_{\lambda} \Gamma(x, \lambda) d x- \\
\int_{\Omega \times \mathbb{R}_{\lambda}} h\left\{\int_{\lambda}^{\infty} \eta(s) d s\right\}\left(\lambda^{\gamma}-\bar{p}\right) d_{\lambda} \Gamma(x, \lambda) d x=(2+\nu) \int_{\Omega \times \mathbb{R}_{\lambda}} h \eta d_{\lambda}(\lambda M(x, \lambda)) d x .
\end{array}
$$

Since $d_{\lambda} \Gamma(x, \cdot)$ is a probability measure and $p(\lambda)=\lambda^{\gamma}$, the function $M$ is non-negative and $\operatorname{Var}{ }_{\lambda} M \leq 2 \bar{p} \in L^{1}(\Omega)$. Substituting (116) into (115) and noting that the linear hull of the set of functions in the form $h \eta$ is dense in $C_{0}^{\infty}(\Omega \times \mathbb{R})$ we obtain (111) which completes the proof.

Renormalization of the kinetic equation Renormalization of kinetic equation (17) is a core of our method. Recall that the notion of a renormalized solution, introduced in pioneering paper [8], plays an important role in the theory of compressible Navier-Stokes equations developed by P.L. Lions and E. Feireisl et al. Moreover, the kinetic equation itself is a result of the renormalization procedure. Formally we can renormalize equation (17) multiplying both its sides by a function $\Psi^{\prime}(\Gamma)$, which leads to the transport equation for a function $\Psi(\Gamma)$. The justification of this construction is a delicate question because we do not know whether the function $\Psi^{\prime}(\Gamma)$ is integrable with respect to the measure $m$ or not. Instead of this property we show that $\Psi^{\prime}(\Gamma)$ satisfies the simple and effective integral inequality. The result is given by the following lemma. Choose an arbitrary $t>1$ and function $\eta \in C^{\infty}(\mathbb{R})$ satisfying the conditions

$$
\eta(\lambda)=1 \text { for } \lambda \leq t-1, \quad \eta(\lambda)=0 \text { for } \lambda \geq t+1, \quad \eta^{\prime}(\lambda) \leq 0
$$

and fix a function $\Psi \in C^{2}(\mathbb{R})$ such that

$$
\Psi^{\prime \prime}(\lambda) \leq 0, \quad \Psi(\lambda) \geq 0 \text { for } \lambda \in[0,1], \quad \Psi(0)=0 .
$$


Lemma 8.2 Under the assumptions of Lemma 8.1 for all $t>2+2\|f\|_{L^{\infty}(\Omega)} / \alpha$,

$$
\begin{gathered}
\int_{\Omega \times[-\infty, t-1)} \Psi(\Gamma) d x d \lambda \leq-(t+1) \int_{\Omega \times \mathbb{R}_{\lambda}} \eta^{\prime}\{\Psi(\Gamma)(|\operatorname{div} \mathbf{u}|+2 \alpha)+\sigma M\} d x d \lambda- \\
\sigma(t+1) \int_{\Omega \times \mathbb{R}_{\lambda}} \eta^{\prime} \lambda^{-1} d m(x, \lambda), \quad \sigma=\max _{[0,1]}\left|\Psi^{\prime}\right| .
\end{gathered}
$$

Proof. Let $\theta^{3}$ and $\theta^{1}$ be regularising kernels in $\mathbb{R}^{3}$ and $\mathbb{R}^{1}$, respectively i.e.

$$
\theta^{j} \in \mathcal{D}_{+}\left(\mathbb{R}^{j}\right), \quad \int_{\mathbb{R}^{j}} \theta^{j}(x) d x=1, \quad \text { spt } \theta^{j} \Subset\{|x| \leq 1\} .
$$

The corresponding mollifiers are defined by the equalities

$$
u_{k, \infty}(x, \lambda)=k^{3} \int_{\Omega} \theta^{3}(k(x-y)) u(y, \lambda) d y, \quad u_{\infty, k}(x, \lambda)=k \int_{\mathbb{R}} \theta^{1}(k(\lambda-\mu)) u(x, \mu) d \mu,
$$

for each $u \in L_{l o c}^{1}(\Omega \times \mathbb{R})$ and dist $(x, \partial \Omega)>k^{-1}$. We will simply write $u_{k, n}$ instead of $\left(u_{k, \infty}\right)_{\infty, n}$. Substituting the test function

$$
\zeta(x, \lambda)=k^{3} n \vartheta^{3}\left(k\left(x_{0}-x\right)\right) \vartheta^{1}\left(n\left(\lambda_{0}-\lambda\right)\right), \quad \operatorname{dist}\left(x_{0}, \partial \Omega\right)>k^{-1}, \quad \lambda_{0} \in \mathbb{R},
$$

into (111) we arrive at the equality

$$
\begin{gathered}
\partial_{\lambda}\left\{\left[(\lambda(\alpha+\operatorname{div} \mathbf{u})-f] \Gamma_{k, n}\right\}-\alpha \Gamma_{k, n}-\operatorname{div}\left(\Gamma_{k, n} \mathbf{u}\right)-\partial_{\lambda}(m+\lambda M)_{k, n}=\right. \\
\left(\mathfrak{r}_{1}\right)_{k, \infty}+\mathfrak{r}_{2}+\partial_{\lambda} \mathfrak{r}_{3} \equiv \mathfrak{r},
\end{gathered}
$$

which holds true in any domain $\Omega^{\prime} \times \mathbb{R}_{\lambda}$ with $\operatorname{dist}\left(\Omega^{\prime}, \partial \Omega\right)<k^{-1}$. Here the remainders $\mathfrak{r}_{j}$ are given by

$$
\begin{gathered}
\mathfrak{r}_{1}=-(\alpha+\operatorname{div} \mathbf{u}) \partial_{\lambda}\left[(\lambda \Gamma)_{\infty, n}-\lambda \Gamma_{\infty, n}\right], \quad \mathfrak{r}_{2}=\operatorname{div}\left[\left(\Gamma_{\infty, n} \mathbf{u}\right)_{k, \infty}-\Gamma_{k, n} \mathbf{u}\right], \\
\mathfrak{r}_{3}=\lambda \Gamma_{k, n} \operatorname{div} \mathbf{u}-\lambda\left(\Gamma_{\infty, n} \operatorname{div} \mathbf{u}\right)_{k, \infty}+\left(\Gamma_{\infty, n} f\right)_{k, \infty}-\Gamma_{k, n} f
\end{gathered}
$$

Recall that $\Gamma_{k, n}$ and $(m+\lambda M)_{k, n}$ are smooth functions in $\Omega^{\prime} \times \mathbb{R}$. Multiplying the both sides of (118) by $\Psi^{\prime}\left(\Gamma_{k, n}\right)$ and noting that $\partial_{\lambda} \Psi^{\prime}\left(\Gamma_{k, n}\right) \leq 0, M, m \geq 0$ we obtain

$$
(\lambda \alpha-f) \partial_{\lambda} \Psi\left(\Gamma_{k, n}\right)-\operatorname{div}_{x, \lambda}\left(\Psi\left(\Gamma_{k, n}\right) \mathbf{w}\right)-\partial_{\lambda}\left(\Psi^{\prime}\left(\Gamma_{k, n}\right)(m+\lambda M)_{k, n}\right) \geq \Psi^{\prime}\left(\Gamma_{k, n}\right) \mathfrak{r} .
$$

Choose an arbitrary non-negative function $h \in C_{0}^{\infty}(\Omega)$ with spt $h \Subset \Omega^{\prime}$. Multiplying the both sides of (119) by $h(x) \eta(\lambda)$ and integrating the result over $\Omega^{\prime} \times \mathbb{R}_{\lambda}$ we arrive at the 
inequality

$$
\begin{gathered}
\int_{\Omega^{\prime} \times \mathbb{R}_{\lambda}} \Psi\left(\Gamma_{k, n}\right) \eta[\alpha h-\nabla h \cdot \mathbf{u}] d x d \lambda+\int_{\Omega^{\prime} \times \mathbb{R}_{\lambda}} h \eta^{\prime} \Psi\left(\Gamma_{k, n}\right)(\lambda \operatorname{div} \mathbf{u}+\lambda \alpha-f) d x d \lambda- \\
\int_{\Omega^{\prime} \times \mathbb{R}_{\lambda}} h \eta^{\prime} \Psi^{\prime}\left(\Gamma_{n, k}\right)(m+\lambda M)_{k, n} d x d \lambda \leq-\int_{\Omega^{\prime} \times \mathbb{R}_{\lambda}} h \eta \Psi^{\prime}\left(\Gamma_{k, n}\right) \mathfrak{r} d x d \lambda .
\end{gathered}
$$

Since $\eta^{\prime} \leq 0$ and $m+\lambda M \geq 0$, we have

$$
\int_{\Omega^{\prime} \times \mathbb{R}_{\lambda}} h \eta^{\prime} \Psi^{\prime}\left(\Gamma_{k, n}\right)(m+\lambda M)_{k, n} d x d \lambda \leq \min _{[0,1]} \Psi^{\prime} \int_{\Omega^{\prime} \times \mathbb{R}_{\lambda}} h \eta^{\prime}(m+\lambda M)_{k, n} d x d \lambda,
$$

which yields

$$
\begin{gathered}
\int_{\Omega^{\prime} \times \mathbb{R}_{\lambda}} \Psi\left(\Gamma_{k, n}\right) \eta[\alpha h-\nabla h \cdot \mathbf{u}] d x d \lambda+\int_{\Omega^{\prime} \times \mathbb{R}_{\lambda}} h \eta^{\prime} \Psi\left(\Gamma_{k, n}\right)(\lambda \operatorname{div} \mathbf{u}+\lambda \alpha-f) d x d \lambda- \\
\min _{[0,1]} \Psi^{\prime} \int_{\Omega^{\prime} \times \mathbb{R}_{\lambda}} h \eta^{\prime}(m+\lambda M)_{k, n} d x d \lambda \leq-\int_{\Omega^{\prime} \times \mathbb{R}_{\lambda}} h \eta \Psi^{\prime}\left(\Gamma_{k, n}\right) \mathfrak{r} d x d \lambda .
\end{gathered}
$$

Now our task is to show that the right side tends to 0 as $k, n \rightarrow \infty$. Fix an arbitrary $n>0$. Since the mapping $(x, \lambda) \rightarrow \mathbf{u}(x)$, which does not depend on $\lambda$, belongs to $L^{\infty}\left(\mathbb{R}_{\lambda}, H^{1}(\Omega)\right)$, it follows from Lemma 2.3 in $\left[8\right.$ that $\mathfrak{r}_{2} \rightarrow 0$ in $L_{l o c}^{1}(\Omega \times \mathbb{R})$ when $k \rightarrow \infty$. Obviously, the sequence $\mathfrak{r}_{3} \rightarrow 0$ converges in $L_{\text {loc }}^{2}(\Omega \times \mathbb{R})$ when $k \rightarrow \infty$. It follows from this that for each fixed $n$,

$$
\lim _{k \rightarrow \infty} \int_{\Omega^{\prime} \times \mathbb{R}_{\lambda}} h \eta \Psi^{\prime}\left(\Gamma_{k, n}\right) \mathfrak{r} d x d \lambda=\int_{\Omega^{\prime} \times \mathbb{R}_{\lambda}} h \Psi^{\prime}\left(\Gamma_{\infty, n}\right) \eta \mathfrak{r}_{1} d x d \lambda .
$$

It is easy to see that

$$
\partial_{\lambda}\left[(\lambda \Gamma)_{\infty, n}-\lambda \Gamma_{\infty, n}\right](x, \lambda)=k \int_{\mathbb{R}} \theta(k(\lambda-\mu)) \Gamma(x, \mu) d \mu, \quad \theta(s)=s \theta^{1^{\prime}}(s)+\theta^{1}(s) .
$$

Since $\theta$ is compactly supported and $\int_{\mathbb{R}} \theta(s) d s=0$, the sequence $\partial_{\lambda}\left[(\lambda \Gamma)_{\infty, n}-\lambda \Gamma_{\infty, n}\right](x, \lambda)$ converges to 0 in $L_{l o c}^{r}(\Omega \times \mathbb{R})$ for each $r<\infty$. Hence $\mathfrak{r}_{1} \rightarrow 0$ in $L_{l o c}^{r}(\Omega \times \mathbb{R})$, which implies

$$
\lim _{n \rightarrow \infty} \lim _{k \rightarrow \infty} \int_{\Omega^{\prime} \times \mathbb{R}_{\lambda}} h \eta \Psi^{\prime}\left(\Gamma_{k, n}\right) \mathfrak{r} d x d \lambda=0 .
$$


Letting $k \rightarrow \infty, n \rightarrow \infty$ in (120) we conclude that the inequality

$$
\begin{gathered}
\int_{\Omega \times[-\infty, t-1)} h \Psi(\Gamma) d x d \lambda-\int_{\Omega \times \mathbb{R}_{\lambda}} \eta \Psi(\Gamma) \nabla h \cdot \mathbf{u} d x d \lambda \leq \\
-(t+1) \int_{\Omega \times \mathbb{R}_{\lambda}} h \eta^{\prime}(\Psi(\Gamma)(|\operatorname{div} \mathbf{u}|+2 \alpha)+M) d x d \lambda-(t+1) \int_{\Omega \times \mathbb{R}_{\lambda}} h \eta^{\prime} \lambda^{-1} d m(x, \lambda) .
\end{gathered}
$$

holds for all $t>2 \alpha^{-1}\|f\|_{L^{\infty}(\Omega)}+2$ and all non-negative $h \in C_{0}^{\infty}(\Omega)$. Now choose a sequence of functions $h_{j} \in C_{0}^{\infty}(\Omega)$ such that $h_{j} \rightarrow 1$ as $j \rightarrow \infty$ in $\Omega$ and $\left|\nabla h_{j}(x)\right| \leq j$ dist $(x, \partial \Omega)$. Substituting $h=h_{j}$ into (111), letting $j \rightarrow \infty$, and noting that $\left\|\nabla h_{j} \cdot \mathbf{u}\right\|_{L^{1}(\Omega)} \rightarrow 0$ we finally obtain (117) which completes the proof

The proof of equality $\Gamma(1-\Gamma)=0$ The last part of the proof is based on the following lemma

Lemma 8.3 Under the above assumptions,

$$
\int_{\mathbb{R}_{\lambda}} \eta^{\prime}(\lambda) M(x, \lambda) d \lambda=-\int_{[0, \infty)} \eta^{\prime \prime}(\lambda) \mathcal{V}_{\lambda}(x) d \lambda,
$$

where $\mathcal{V}_{\lambda}$ is defined by (107).

Proof. It is easy to see that

$$
\begin{gathered}
-(2+\nu) \int_{\mathbb{R}_{\lambda}} \eta^{\prime}(\lambda) M(x, \lambda) d \lambda=\iint_{[0, \infty)}\left(\int_{[\lambda, \infty)} \eta^{\prime \prime}(s) d s\right)\left(\int_{[\lambda, \infty)}\left(t^{\gamma}-\bar{p}\right) d_{t} \Gamma(x, t)\right) d \lambda= \\
\int_{[0, \infty)} \eta^{\prime \prime}(s)\left(\int_{[0, s)} d \lambda \int_{[\lambda, \infty)}\left(t^{\gamma}-\bar{p}\right) d_{t} \Gamma(x, t)\right) d s=\int_{[0, \infty)} \eta^{\prime \prime}(s)\left(\int_{[0, \infty)} \min \{t, s\}\left(t^{\gamma}-\bar{p}\right) d_{t} \Gamma(x, t)\right) d s= \\
\left.\int_{[0, \infty)} \eta^{\prime \prime}(s)\left(\frac{\min \{\varrho, s\} p}{\min \{\varrho, s\}} \bar{p}\right)\right) d s .
\end{gathered}
$$

On the other hand, Lemma $\overline{7.3}$ yields $\overline{\min \{\varrho, \lambda\} p}-\overline{\min \{\varrho, \lambda\}} \bar{p}=(2+\nu) \mathcal{V}_{\lambda}(x)$, and the lemma follows .

Take $\Psi$ in the simplest form $\Psi(\Gamma)=\Gamma(1-\Gamma)$ with $\sigma=1$. Since $\eta^{\prime}(\lambda)$ vanishes for $\lambda<1$, we have

$$
\begin{gathered}
-\int_{\Omega \times \mathbb{R}_{\lambda}} \eta^{\prime} \Psi(\Gamma)(|\operatorname{div} \mathbf{u}|+2 \alpha) d x d \lambda=\int_{[1, \infty)} \eta^{\prime \prime}(\lambda) d \lambda \int_{\Omega \times[1, \lambda)} \Psi(\Gamma(x, s))(|\operatorname{div} \mathbf{u}(x)|+2 \alpha) d x d s \\
-\int_{\Omega \times \mathbb{R}_{\lambda}} \eta^{\prime} \lambda^{-1} d m(x, \lambda)=\int_{[1, \infty)} \eta^{\prime \prime}(\lambda) d \lambda \int_{\Omega \times[1, \lambda)} s^{-1} d m(x, s)
\end{gathered}
$$


Substituting these identities along with (122) into (117) implies the inequality

$$
\int_{\Omega \times[-\infty, t-1)} \Psi(\Gamma) d x d \lambda \leq(1+t) \int_{[1, \infty)} \eta^{\prime \prime}(\lambda) \wp(\lambda) d \lambda,
$$

in which the function $\wp:[1, \infty) \mapsto \mathbb{R}$ is given by

$$
\wp(\lambda)=\int_{\Omega \times[1, \lambda)} \Psi(\Gamma(x, s))(|\operatorname{div} \mathbf{u}(x)|+2 \alpha) d x d s+\int_{\Omega} \mathcal{V}_{\lambda}(x) d x+\int_{\Omega \times[1, \lambda)} s^{-1} d m(x, s) .
$$

It follows from Lemma 7.5 that for $\lambda>1$,

$$
|\wp(\lambda)| \leq c\left(1+\|\mathbf{u}\|_{H^{1,2}(\Omega)}\right)\|\mathfrak{H}\|_{L^{2}(\Omega)}+\left\|\mathcal{V}_{\lambda}\right\|_{L^{1}(\Omega)}+c \int_{\Omega \times[1, \infty)}(1+\lambda)^{-1} d m(x, \lambda) \leq c,
$$

where a constant $c$ does not depend on $\lambda$. Next set $\eta(\lambda)=\int_{\lambda}^{\infty} \omega(s-t) d s$, where $\omega$ is a smooth, even, non-negative function supported on the interval $(-1,1)$ and such that $\int_{\mathbb{R}} \omega(s) d s=1$. Then inequality (123) can be rewritten in the form

$$
\int_{\Omega \times[-\infty, t-1)} \Psi(\Gamma) d x d \lambda \leq(1+t) \frac{d}{d t}(\omega * \wp)(t),
$$

Since the smooth function $(\omega * \wp)(t)$ is uniformly bounded on the interval $[1, \infty)$, then there is a sequence $t_{k} \rightarrow \infty$ such that $\lim _{k \rightarrow \infty}\left(t_{k}+1\right) \frac{d}{d t}(\omega * \wp)\left(t_{k}\right) \leq 0$. Substituting $t=t_{k}$ into (124) and letting $k \rightarrow \infty$ we conclude that $\Psi(\Gamma)=0$ a.e in $\Omega \times \mathbb{R}_{\lambda}$, which completes the proof of Theorem 6.5

\section{References}

[1] D. Adams, Arkiv for Matematik 14, (1976) 125-140.

[2] D. Adams and L. Hedberg Function Spaces and Potential Theory ( Springer-Verlag, Berlin etc 1995).

[3] C. Agmon, A. Douglis, L. Nirenberg, Comm. Pure Apll. Math. 12, (1959) 623-727.

[4] W. K. Allard, Ann. Math. 95(1972) 417-491.

[5] J. M. Ball. A version of the fundamental theorem for Young measures, in PDEs and continuum Models of Phase Trans., Lecture Notes in Physics, 344(1989) 241-259.

[6] G. Q. Chen, B. Perthame, Ann. Inst. H. Poincare Anal Nonlineaire 20, (2003) 645-668. 
[7] M.G. Crandall, L. Tartar, Proc. AMS 78, (1980) 385-390

[8] R. J. DiPerna, P. L. Lions, Invent. Math. 48,(1989) 511-547.

[9] R. J. DiPerna, A. J. Majda, Comm. Math. Phys. 108,(1987) 667-689.

[10] R. J. DiPerna, A. J. Majda, J. Am. Math. Soc. 1,(1988) 59-95.

[11] H. Federer, Geometric measure theory, (Springer-Verlag, New-York etc. 1969)

[12] E. Feireisl, Comment. Math. Univ. Carolinae 42,(2001) 83-98.

[13] E. Feireisl, Š. Matušů-Nečasová, H. Petzeltová, I. Straškraba, Arch. Rational Mech. Anal.149, (1999) 69-96.

[14] E. Feireisl, A.H. Novotný, H. Petzeltová, J. of Math. Fluid Mech. 3, (2001), 358-392.

[15] E. Feireisl Dynamics of Viscous Compressible Fluids ( Oxford University Press, Oxford 2004)

[16] D. Gilbarg and N. S. Trudinger Elliptic Partial Differential Equations of Second Order ( Springer-Verlag, Berlin etc 1983).

[17] M.A. Krasnoselsky, Positive Solutions to Operator Equations (Fizmatgiz, Moscow 1962)(in Russian).

[18] S. G. Krejn (ed), N. Ya. Vilenkin, E. A. Gorin, A. G. Kosruchenko, M. A. Krasnosel'skij, V. P. Maslov, B. S. Mityagin, Yu. I. Petunin, Ya. B. Rutirskij, V.I. Sobolev, V. Ya. Stetsenko, L. D. Faddeev, E. S. Tsitlanadze, Functional Analysis ( WoltersNoordhoff Publishing, Groningen, 1972)

[19] P. L. Lions, Mathematical topics in fluid dynamics, Vol. 2, Compressible models ( Oxford Science Publication, Oxford 1998)

[20] P. L. Lions, On some chalenging problems in nonlinear partial differential equations in V. Arnold (ed) et al., Mathematics: Frontiers and perspectives ( American Mathematical Society, Providence 2000) 121-135.

[21] V. G. Maz’ja, Izv. Akad. Nauk SSSR, Ser. Mat. 28, (1964) 1145-1172.

[22] V. G. Maz'ja Sobolev Spaces, (Springer-Verlag Berlin etc. 1985)

[23] M. Padula, Arch. Ration. Mech. Anal.97, (1987) 89-102.

[24] M. Padula, Steady Flows of Barotropic Viscous Fluids. In : Classical Problems in Mechanics, ed. R. Russo, 253-345, Quad. Mat., 1, Aracne, Rome, 1997.

[25] P.I. Plotnikov, J. Sokolowski (Les prepublications de l' Institut Elie Cartan No. 35, Nancy 2002, 37 pages) to appear in : Journal of Mathematical Fluid Mechanics.

RR $n^{\circ} 5481$ 
[26] W. P. Ziemer, Weakly differntiable functions ( Springer-Verlag, New-York etc. 1989)

[27] Y. Zheng, Comm. Math. Phys. 135, (1991) 581-594. 


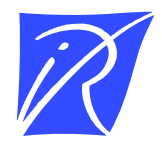

\section{Unité de recherche INRIA Sophia Antipolis 2004, route des Lucioles - BP 93 - 06902 Sophia Antipolis Cedex (France)}

Unité de recherche INRIA Futurs : Parc Club Orsay Université - ZAC des Vignes 4, rue Jacques Monod - 91893 ORSAY Cedex (France)

Unité de recherche INRIA Lorraine : LORIA, Technopôle de Nancy-Brabois - Campus scientifique 615, rue du Jardin Botanique - BP 101 - 54602 Villers-lès-Nancy Cedex (France)

Unité de recherche INRIA Rennes : IRISA, Campus universitaire de Beaulieu - 35042 Rennes Cedex (France)

Unité de recherche INRIA Rhône-Alpes : 655, avenue de l'Europe - 38334 Montbonnot Saint-Ismier (France)

Unité de recherche INRIA Rocquencourt : Domaine de Voluceau - Rocquencourt - BP 105 - 78153 Le Chesnay Cedex (France) 